\title{
ROSENY DOS REIS RODRIGUES
}

Avaliação da aeração e edema pulmonar por meio de tomografia computadorizada em pacientes submetidos a revascularização do miocárdio

Tese apresentada à Faculdade de Medicina da Universidade de São Paulo para obtenção do título de Doutor em Ciências

Programa de: Anestesiologia

Orientador: Dr. Luiz Marcelo Sá Malbouisson

São Paulo 
Dados Internacionais de Catalogação na Publicação (CIP)

Preparada pela Biblioteca da

Faculdade de Medicina da Universidade de São Paulo

Creprodução autorizada pelo autor

Rodrigues, Roseny dos Reis

Avaliação da aeração e edema pulmonar por meio de tomografia computadorizada em pacientes submetidos a revascularização do miocárdio / Roseny do Reis Rodrigues. -- São Paulo, 2010.

Tese(doutorado)--Faculdade de Medicina da Universidade de São Paulo. Programa de Anestesiologia.

Orientador: Luiz Marcelo Sá Malbouisson.

Descritores: 1.Atelectasia pulmonar 2.Tomografia computadorizada helicoidal 3.Cirurgia cardíaca 4.Hipoxemia

USP/FM/DBD-237/10 
DEDICATÓRIA 
Ao meu esposo, Wilson, pela paciência e incentivo nesta jornada.

Aos meus pais, por compreenderem minhas ausências.

A Deus, por permitir a conclusão deste trabalho. 
AgRadecimentos

$\overline{=}$ 
Ao meu amigo e orientador, Dr. Luiz Marcelo Sá Malbouisson, por ter apontado o caminho da ciência e a quem devo grande parte de minha formação científica.

Ao Prof. Dr. José Otávio Costa Auler Júnior, por me proporcionar inúmeras oportunidades ao longo da vida profissional.

A Profa. Dra. Maria José Carmona, por ser exemplo de médica, professora, mãe, conseguindo desempenhar todas as funções com sucesso, pelo estímulo à pesquisa clínica.

Aos colegas Dra. Kátia Beck Le Bihan, Dr. Luiz Guilherme, Dra. Luciana Moraes e Dra. Marilde Albuquerque, pela colaboração neste projeto.

À equipe da radiologia do Instituto do Coração, pela valiosa ajuda durante a fase de coleta de dados.

Aos colegas do Serviço de Anestesiologia e Terapia Intensiva Cirúrgica do InCor - HCFMUSP, pela ajuda nas diversas fases deste estudo e auxílio em todas as horas.

Aos pacientes e seus familiares, por entenderam o objetivo desta pesquisa e autorizarem sua realização. 
SUMÁRIO 


\section{SUMÁRIO}

Lista de Abreviaturas e Siglas

Lista de Tabelas

Lista de Figuras

Resumo

Summary

1. INTRODUÇÃO.

2. OBJETIVOS

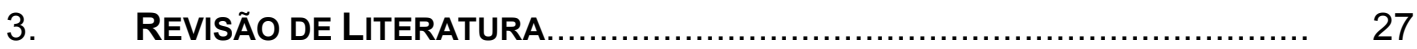

3.1. Avaliação pulmonar com tomografia computadorizada....................... 28

3.1.1 Contribuições da tomografia computadorizada na avaliação pulmonar de pacientes com insuficiência respiratória.

3.1.2 Contribuição da TC para avaliação pulmonar em pacientes submetidos à anestesia geral.

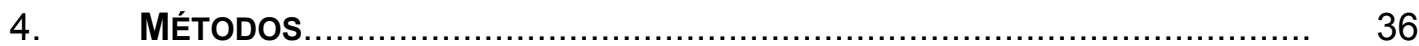

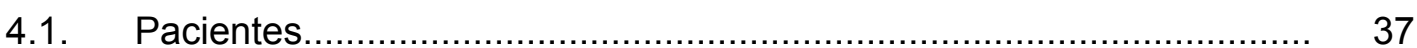

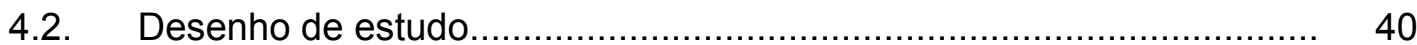

4.3. Técnica anestésica, monitorização e cuidados pós-operatórios........... 42

4.4. Protocolo de estudo por tomografia computadorizada........................ 45

4.4.1 Aquisição das tomografias computadorizadas.................................... 45

4.4.2 Avaliação do volume pulmonar de gás, tecido e total.......................... 46

4.4.3 Análise da aeração pulmonar de acordo com a distribuição do coeficiente CT.............................................................................. 49

4.5. Análise estatística...................................................................... 50

4.5.1 Cálculo amostral............................................................................. 50 
5. Resultados.

5.1. Dados hemodinâmicos e trocas gasosas durante o período de estudo

5.2. Avaliação pulmonar pré e pós-operatória por meio de tomografia computadorizada

5.3. Correlações entre as alterações observadas na morfologia pulmonar, trocas gasosas e duração da circulação extracorpórea.

6. DIscUSSÃo

7. Conclusões.

8. REFERÊNCIAS 
Lista de AbReViaturas e Siglas 


\section{Lista DE AbReViaturas e Siglas}

\begin{tabular}{|c|c|}
\hline ACP & Analgesia controlada pelo paciente \\
\hline ASA & American Society Anesthesia \\
\hline Bpm & Batimento por minuto \\
\hline CEC & Circulação extracorpórea \\
\hline CRF & Capacidade residual funcional \\
\hline $\mathrm{CM}^{2}$ & Centímetros quadrados \\
\hline $\mathrm{CO}_{2}$ & Gás carbônico \\
\hline DPOC & Doença pulmonar obstrutiva crônica \\
\hline ECG & Eletrocardiograma \\
\hline $\mathrm{Fc}$ & Frequência cardíaca \\
\hline $\mathrm{FiO}_{2}$ & Fração inspirada de oxigênio \\
\hline FEVE & Fração de ejeção do ventrículo esquerdo \\
\hline FAPESP & Fundação de Amparo à Pesquisa do Estado de São Paulo \\
\hline G & Grama \\
\hline Ipm & Inspirações por minuto \\
\hline $\mathrm{H}$ & Hora(s) \\
\hline $\mathrm{H}_{2} \mathrm{O}$ & Água \\
\hline IMC & Índice de massa corpórea \\
\hline IC & Índice Cardíaco \\
\hline IS & Índice Sistólico \\
\hline IRVS & Índice de Resistência vascular sistêmica \\
\hline IRVP & Índice de resistência vascular pulmonar \\
\hline $\mathrm{Kv}$ & Kilovolt \\
\hline $\mathrm{Kg}$ & Kilograma \\
\hline Mas & Miliamperes \\
\hline Min. & Minuto \\
\hline$\mu g$ & Microgramas \\
\hline MG & Miligramas \\
\hline ML & Mililitros \\
\hline $\mathrm{MmHg}$ & Milímetros de mercúrio \\
\hline PAM & Pressão Arterial Média \\
\hline
\end{tabular}




$\begin{array}{ll}\mathrm{PAPm} & \text { Pressão de Artéria Pulmonar Média } \\ \mathrm{PaO}_{2} & \text { Pressão parcial arterial de oxigênio } \\ \mathrm{PaCO}_{2} & \text { Pressão parcial arterial de gás carbônico } \\ \mathrm{PvCO}_{2} & \text { Pressão parcial venosa de gás carbônico } \\ \mathrm{PEEP} & \text { Pressão positiva expiratória final } \\ \mathrm{PVC} & \text { Pressão venosa central } \\ \mathrm{POAP} & \text { Pressão de Oclusão de Artéria Pulmonar } \\ \mathrm{RM} & \text { Revascularização miocárdica } \\ \mathrm{SaO} & \\ \mathrm{SvO} & \text { Saturação arterial de oxigênio } \\ \mathrm{SARA} & \text { Saturação venosa de oxigênio } \\ \mathrm{TC} & \text { Síndrome da Angústia Respiratória do Adulto } \\ \mathrm{UH} & \text { Tomografia Computadorizada } \\ \mathrm{UI} & \text { Unidades Hounsfield } \\ \mathrm{UTI} & \text { Unidade Internacional } \\ \text { USP } & \text { Unidade de terapia intensiva } \\ \mathrm{TCLE} & \text { Universidade de São Paulo } \\ \mathrm{VPH} & \text { Termo de consentimento Livre e Esclarecido } \\ \mathrm{VNI} & \text { Vasoconstricção pulmonar hipóxica } \\ & \text { Ventilação não-invasiva }\end{array}$


Lista DE TABELAS 


\section{LISTA DE TABELAS}

Tabela I

Tabela II

Tabela III

Tabela IV
Dados demográficos e clínicos dos pacientes estudados.

Dados relacionados ao tempo de CEC, duração da cirurgia, volume total de diurese e volume infundido no intraoperatório.

Dados hemodinâmicos durante a cirurgia e nas primeiras 24 horas pós-operatórias.

Perfil metabólico durante a cirurgia e nas primeiras 48 horas pós-operatórias 
Lista DE FIGURAS

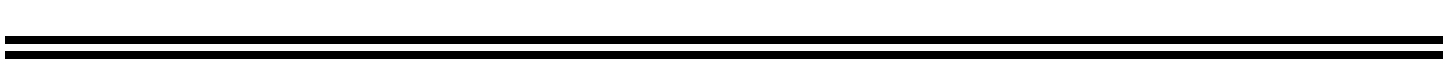




\section{LISTA DE FIGURAS}

Figura 1 - $\quad$ Fluxograma do número total de pacientes............................ 39

Figura 2 - $\quad$ Cronograma do estudo................................................... 40

Figura 3 - $\quad$ Comportamento da relação $\mathrm{PaO}_{2} / \mathrm{FiO}_{2}$ durante o estudo.... 59

Figura 4 - Imagens de TC na altura do ápice, hilo vascular pulmonar e $2 \mathrm{~cm}$ acima do diafragma obtidas no pré-operatório e o primeiro dia pós-operatório................................................ 60

Figura 5 - Volume pulmonar total, de tecido e gás computados em reconstruções volumétricas a partir de tomografias computadorizadas de pulmão inteiro no pré-operatório e no pós-operatório.

Figura 6 - Figura demonstrando aumento significativo do peso total do parênquima pulmonar no período pós-operatório de $19 \%(p<0,001)$.

Figura 7 - Correlação entre a variação percentual da fração de gás (redução percentual da CRF) e a variação percentual do parênquima pulmonar com aeração reduzida. 
RESUMO 
Rodrigues RR. Avaliação da aeração e edema pulmonar por meio de tomografia computadorizada em pacientes submetidos a revascularização do miocárdio [tese]. São Paulo: Faculdade de Medicina, Universidade de São Paulo; 2010.

Introdução: A disfunção respiratória é uma das complicações de maior prevalência no período pós-operatório de pacientes submetidos à cirurgia de revascularização do miocárdio (RM) com circulação extracorpórea (CEC), mesmo na ausência de doença pulmonar prévia ${ }^{1}$. Por meio de tomografia computadorizada, foram investigadas as alterações pulmonares pósoperatórias e o seu impacto na oxigenação. Métodos: vinte pacientes não hipoxêmicos em programação de cirurgia eletiva de revascularização do miocárdio com CEC foram estudados. Medidas hemodinâmicas, e amostras sanguíneas seriadas foram obtidas antes da cirurgia, após a intubação orotraqueal, após a CEC, na UTI, $12 \mathrm{~h}, 24 \mathrm{~h}$ e $48 \mathrm{~h}$ após a cirurgia. Tomografias volumétricas pré e pós-operatórias foram adquiridas em condições de apneia após uma expiração espontânea. Os dados foram analisados usando teste de t Student; o comportamento temporal dos dados hemodinâmicos e outras variáveis fisiológicas foi analisado ao longo do tempo, usando análise de variância de uma via para repetidas medidas, seguido pelo teste de Student-Neumann-Keuls, quando necessário.Resultados: a relação $\mathrm{PaO}_{2} / \mathrm{FiO}_{2}$ diminuiu de forma significativa após a indução da anestesia, atingindo o seu nadir após a saída de CEC. Comparando-se com a TC pré-operatória, foi observada uma redução de $31 \%$ no volume de gás pulmonar $(p<0,001)$, ao passo que foi observado um aumento do volume de tecido de $19 \%(p<0,001)$. A área não aerada dos pulmões aumentou de $253 \pm 97 \mathrm{~g}(\mathrm{p}<0.001)$, de $3 \%$ a $27 \%$, após a cirurgia; e a área pobremente aerada pulmonar apresentou aumento de $72 \pm 68 \mathrm{~g} \mathrm{(p}$ $<0.001$ ), de $24 \%$ to $27 \%$, enquanto a área normalmente aerada pulmonar reduziu de $147 \pm 119 \mathrm{~g}$ ( $p<0.001)$, que representa $72 \%$ to $46 \%$. Não foram observadas correlações entre a relação $\mathrm{PO}_{2} / \mathrm{FIO}_{2}$ ou na fração de shunt nas primeiras 24 horas pós-operatórias Conclusões: A estrutura pulmonar está profundamente modificada após a cirurgia de RM com CEC. Associadas a outros fatores, essas alterações são responsáveis pela ocorrência de hipoxemia resultantes de atelectasias.

Descritores: atelectasia pulmonar, tomografia computadorizada helicoidal, cirurgia cardíaca, hipoxemia 
SUMMARY

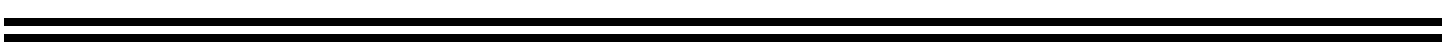


Rodrigues RR. Evaluation of the aeration and edema pulmonary by means of computed tomography in patients undergoing coronary artery bypass graft [tese]. São Paulo: Faculdade de Medicina, Universidade de São Paulo; 2010.

Introduction: Hypoxemia is a frequent complication after coronary artery bypass graft (CABG) with cardiopulmonary bypass (CPB), usually attributed to atelectasis. Using computed tomography (CT), we investigated pulmonary alterations postoperatively and its impact on oxygenation. Methods: Twenty non-hypoxemic patients with normal cardiac function scheduled to CABG under CPB were studied. Hemodynamic measurements and blood samples were obtained before surgery, after intubation, after CPB, at ICU admission, $12 \mathrm{~h}, 24 \mathrm{~h}$ and $48 \mathrm{~h}$ after surgery. Pre and postoperative volumetric thoracic CT scans were acquired in apnea conditions after a spontaneous expiration. Data was analyzed using paired Student $t$ test and two-way repeated measures analysis of variance followed by SNK test when indicated. Results: $\mathrm{PaO}_{2} / \mathrm{FiO}_{2}$ ratio was significantly reduced after anesthesia induction, reaching its nadir after CPB and partially ameliorating $12 \mathrm{~h}$ after surgery. Compared to preoperative CT, there was a postoperative $31 \%$ reduction in pulmonary gas volume $(p<0.001)$ while tissue volume increased by $19 \%(p<0.001)$. Non-aerated lung increased by $253 \pm 97 \mathrm{~g}(\mathrm{p}<0.001)$, from $3 \%$ to $27 \%$, after surgery and poorly-aerated lung by $72 \pm 68 \mathrm{~g}(\mathrm{p}<$ 0.001 ), from $24 \%$ to $27 \%$ while normally-aerated lung was reduced by $147 \pm$ $119 \mathrm{~g}$ ( $p<0.001$ ), from $72 \%$ to $46 \%$. No correlations were observed between $\mathrm{PaO}_{2} / \mathrm{FiO}_{2}$ ratio or shunt fraction at $24 \mathrm{~h}$ postoperatively and postoperative lung alterations. Conclusions: Lung structure is profoundly modified after CABG with CPB. Taken together, these multiple alterations occurring in the lungs are responsible for postoperative hypoxemia instead of atelectasis alone.

Descriptors: pulmomary atelectasis; tomography helicoidal; cardiac surgery; Hipoxemia 
INTRODUÇÃO

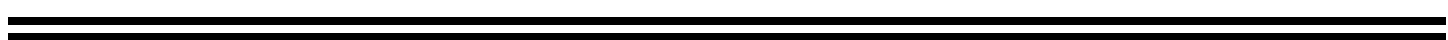




\section{INTRODUÇÃO}

A disfunção respiratória é uma das complicações de maior prevalência no período pós-operatório de pacientes submetidos à cirurgia de revascularização do miocárdio (RM) com circulação extracorpórea (CEC), mesmo na ausência de doença pulmonar prévia ${ }^{1}$. Esta entidade é conhecida por cirurgiões cardíacos, anestesiologistas e intensivistas desde os primórdios da cirurgia cardíaca ${ }^{2}$. A magnitude dos distúrbios respiratórios manifestados no pós-operatório varia de alterações funcionais subclínicas, que ocorrem em uma fração considerável dos pacientes, até a síndrome de angústia respiratória do adulto (SARA), que ocorre em $2 \%$ dos casos após CEC ${ }^{3}$. Em uma série de 461 pacientes consecutivos submetidos à RM no Instituto do Coração do Hospital das Clínicas da Faculdade de Medicina da Universidade de São Paulo, foi observado que $54,2 \%$ dos pacientes apresentaram a relação $\mathrm{PO}_{2} / \mathrm{FiO}_{2}$ menor que $200{ }^{4}$.

Atelectasias, levando a aumento da fração de shunt, são consideradas o principal mecanismo causador de hipoxemia pós-operatória 2. O colapso pulmonar desenvolve-se logo após a indução da anestesia e pode persistir por até alguns dias no período de recuperação pós-operatória $3,5,6,7$. Estima-se que 50 a $90 \%$ dos pacientes submetidos à cirurgia cardíaca apresentem atelectasias no período pós-operatório ${ }^{8,9,10}$. Diferentes mecanismos têm sido propostos para explicar esta incidência: a) deslocamento cefálico do diafragma após o relaxamento muscular pela 
pressão do conteúdo abdominal ${ }^{11}$; b) resposta inflamatória sistêmica induzida pela CEC, que leva a aumento da permeabilidade vascular com consequente edema intersticial pulmonar. Como consequência do edema intersticial, ocorre aumento do peso do parênquima pulmonar, promovendo compressão do parênquima subjacente ${ }^{12}$; c) manipulação cirúrgica intratorácica e d) uso de frações inspiradas de oxigênio em concentrações elevadas na indução anestésica e no perioperatório ${ }^{13}$.

Do ponto de vista fisiopatológico, as consequências imediatas da perda de aeração pulmonar e do edema pulmonar são aumento da mistura venosa de sangue oxigenado na artéria pulmonar, com consequente hipoxemia ${ }^{14,15}$. Isto contribui para o aumento do tempo em ventilação mecânica e maior necessidade de cuidados fisioterápicos no pós-operatório 16. Na circulação pulmonar, devido à vasoconstrição pulmonar hipóxica, pode ocorrer elevação das pressões da artéria pulmonar ${ }^{17}$ e disfunção cardíaca direita em alguns pacientes ${ }^{18}$. Além disso, considera-se que o parênquima pulmonar colapsado pode possibilitar a formação de sítios propícios para o desenvolvimento de infecções.

Analisando imagens justadiafragmáticas de tomografia computadorizada (TC) torácica, alguns investigadores observaram que o colapso pulmonar pós-operatório pode comprometer até $20 \%$ da área dos pulmões ${ }^{10,19}$. Estas atelectasias distribuem-se principalmente nas regiões pulmonares dependentes ${ }^{20}$. Contudo, até o presente momento, nenhum estudo quantificou o volume total e a massa das atelectasias pulmonares pós-operatórias que ocorrem em todo o pulmão. Conforme a técnica descrita 
por Puybasset et $a^{21}$, é possível, utilizando tomografia computadorizada helicoidal, quantificar os volumes pulmonares de gás e tecido (parênquima, sangue e água extravascular pulmonar) e computar a quantidade de parênquima pulmonar hiperinsuflado, definidos como compartimentos pulmonares com densidade radiológica $<-900$ unidades Hounsfield $(\mathrm{UH})$, normalmente aerado $(-900$ a $-500 \mathrm{UH})$, pouco aerado $(-500$ a $-100 \mathrm{HU})$ e atelectasiado (> -100 UH) (18-20). Por meio desta técnica, é possível quantificar os efeitos combinados da anestesia, manipulação cirúrgica e circulação extracorpórea sobre a perda de aeração na distribuição de gás e tecido em todo o pulmão.

Além da avaliação da quantidade de gás, a análise de tomografias computadorizadas volumétricas permite a quantificação do componente de tecido pulmonar, que engloba o parênquima pulmonar propriamente dito, os fluidos fisiológicos intrapulmonares (sangue e linfa) e a água extravascular pulmonar. Comparando tomografias pré e pós-operatórias, é também possível quantificar a variação do volume e peso do tecido pulmonar, que, em última instância, reflete o aparecimento de edema pulmonar.

Este estudo foi desenhado para avaliar as alterações na morfologia do parênquima pulmonar após cirurgia de revascularização do miocárdio com CEC, e não apenas em uma região próxima ao diafragma ou em regiões supostamente representativas de todo o pulmão, por meio de análise de tomografias computadorizadas volumétricos de tórax realizados em pacientes submetidos a cirurgia cardíaca. 


\section{OBJetivos}




\section{OBJetivos}

O objetivo principal deste estudo foi:

1) Avaliar as alterações na morfologia do parênquima pulmonar que ocorrem após cirurgia de revascularização do miocárdio com CEC.

Os objetivos secundários foram:

2) Quantificar o edema pulmonar pós-operatório (água extravascular pulmonar);

3) Correlacionar as alterações na morfologia pulmonar com alterações nas trocas gasosas. 
REVISÃO DE LITERATURA

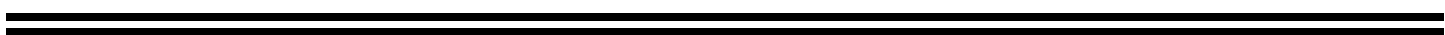




\section{REVISÃo de LITERATURA}

Tem-se demonstrado, de forma consistente, que pacientes submetidos a cirurgia cardíaca com circulação extracorpórea apresentam frequentemente disfunção pulmonar de graus variáveis, cujas causas não são completamente esclarecidas ${ }^{22,23}$. Dentre as manifestações clínicas da disfunção pulmonar pós-operatória, a hipoxemia é um evento bastante comum, mesmo em pacientes com função cardiopulmonar prévia normal no contexto do perioperatório ${ }^{24,25}$ e a formação de atelectasias tem sido apontada como principal causa. Os métodos de imagem à beira do leito são poucos sensíveis e específicos para avaliação das alterações morfológicas pulmonares pós-operatórias, em comparação com a avaliação pulmonar por tomografia computadorizada.

Esta revisão de literatura discorrerá sobre o uso da tomografia computadorizada como método de avaliação pulmonar.

\subsection{Avaliação pulmonar com tomografia computadorizada}

Desde o início dos anos 80, a tomografia computadorizada (TC) de tórax tem sido utilizada para avaliar o comprometimento do parênquima pulmonar. A introdução da TC de tórax representou um grande salto qualitativo em relação à radiografia convencional de tórax na análise dos pulmões de pacientes com insuficiência respiratória aguda, como decorrência de dois atributos fundamentais: 1) resolução do problema da 
superposição de estruturas inerente à radiografia de tórax e 2) aumento da definição das estruturas anatômicas, isto é, 0,5\% de alteração na atenuação aos raios $\mathrm{X}$ podem ser detectados pela $\mathrm{TC}$, enquanto para a radiografia convencional de tórax é necessária uma alteração de $10 \%$ na atenuação aos raios $\mathrm{X}$.

Em 1982, Breiman e colaboradores descreveram uma técnica que possibilitava a determinação de maneira não-invasiva e acurada do volume de estruturas corporais in vivo, a partir de imagens contíguas obtidas através de tomografia computadorizada ${ }^{26}$. Nesse estudo, imagens tomográficas de $1 \mathrm{~cm}$ e $2 \mathrm{~cm}$ de espessura foram obtidas de fantasmas para calibração,de rins caninos e baços humanos antes da esplenectomia. O volume total de determinada estrutura foi obtido somando-se os volumes parciais das áreas de interesse delineados nas imagens tomográficas contíguas de onde a estrutura aparecia. O cálculo do volume das diversas estruturas através da tomografia computadorizada foi comparado à mensuração do volume determinado pelo deslocamento da água. A percentagem média de erro do cálculo de volume através da TC foi de $4,95 \%$ para os fantasmas de calibração, 3,86\% para os rins caninos e 3,59\% para baços humanos com cortes tomográficos contíguos com $1 \mathrm{~cm}$ de espessura e $3,65 \%$ com $2 \mathrm{~cm}$ de espessura. A partir destes resultados iniciais, a TC começou a ser utilizada para análise volumétrica de estruturas corporais in vivo. As dificuldades no reconhecimento visual e no contorno das áreas de interesse pareciam ser para os autores fontes de erro mais significantes que os fatores relacionados às estruturas estudadas. 
Com a introdução dos equipamentos de tomografia computadorizada helicoidal de segunda geração na prática clínica, com velocidades de aquisição de imagem muito superiores à geração anterior, tornou-se possível estudar as alterações morfológicas e estruturais póscirúrgicas em todo o pulmão ${ }^{27}$. Utilizando este método de avaliação quantitativa do parênquima pulmonar inteiro através de TC helicoidal, Puybasset e colaboradores, em 1998, descreveram uma perda do volume pulmonar total em pacientes com SARA em torno de $27 \%$ quando comparado a voluntários sadios ${ }^{21}$.

De acordo com Tagliabue e colaboradores, que compararam o impacto do uso de TC de tórax com a radiografia de tórax convencional no curso da avaliação diagnóstica de pacientes com insuficiência respiratória aguda, foi observado que a tomografia computadorizada forneceu informações complementares em $22 \%$ dos casos, influenciando diretamente o curso do tratamento ${ }^{27}$.

Por estes motivos citados acima, a visualização dos cortes pulmonares em toda a extensão dos pulmões é de fundamental importância para a melhor compreensão dos mecanismos de colapso e hipoxemia em pacientes com disfunção pulmonar de qualquer origem ${ }^{28}$.

\subsubsection{Contribuições da tomografia computadorizada na avaliação pulmonar de pacientes com insuficiência respiratória}

A tomografia computadorizada, por sua superior definição de imagem, capacidade de diferenciação de estruturas intratorácicas e 
possibilidade de análise de dados quantitativos gerados pelos algoritmos de análise computadorizada, tem sido amplamente utilizada como ferramenta para o entendimento das alterações pulmonares em diversas situações clínicas e, em particular, na insuficiência respiratória.

Em 1988, Gattinoni e colaboradores ${ }^{29}$ estudaram 20 pacientes com insuficiência respiratória aguda submetidos à CT de tórax em ventilação mecânica com PEEP de 5, 10 e $15 \mathrm{cmH}_{2} \mathrm{O}$, com o objetivo de investigar as relações entre a morfologia e a função pulmonar. Estes autores observaram que as densidades pulmonares concentravam-se basicamente nas regiões dependentes do pulmão. Ainda segundo eles, o peso do tecido pulmonar estava aumentado em duas vezes e o edema pulmonar de alta permeabilidade, descrito como excesso de peso do tecido pulmonar, estava correlacionado à pressão média da artéria pulmonar. O shunt pulmonar estava correlacionado ao volume de parênquima pulmonar não inflado. $\mathrm{O}$ aumento dos níveis de PEEP causou desaparecimento progressivo das densidades radiológicas e aumentou a massa de tecido pulmonar normalmente inflado, enquanto o shunt pulmonar foi reduzido. Os autores concluíram neste estudo que as densidades pulmonares e as eficiências da troca de oxigênio estavam correlacionadas e o efeito principal do aumento da PEEP era o de recrutar as unidades alveolares perfundidas que estavam previamente colapsadas.

Em 1991, Gattinoni e colaboradores descreveram a influência do posicionamento corporal na distribuição das densidades pulmonares em pacientes com insuficiência respiratória aguda ${ }^{30}$. Dez pacientes foram 
submetidos à TC de tórax em posição supina e prona e cada corte tomográfico foi dividido em 10 níveis da região dorsal para a região ventral, constituindo cada nível $10 \%$ da altura do pulmão. Foi observado que, em posição supina, o excesso de peso do tecido pulmonar distribuía-se independentemente da gravidade e de maneira homogênea no parênquima pulmonar. Contudo, o coeficiente CT médio de cada nível aumentava em direção à região dependente do pulmão, sugerindo deflação dos alvéolos ao longo de um eixo vertical. Achados similares foram observados na posição prona, concluindo-se que o pulmão de pacientes com insuficiência respiratória aguda comporta-se como uma estrutura elástica com a massa difusamente aumentada e as regiões dependentes comprimidas pelas estruturas sobrejacentes $^{30}$.

Estes estudos possibilitaram o entendimento do comportamento da distribuição da aeração e colapso pulmonar, que foi posteriormente traduzido no conceito "pulmão de bebê", proposto por Gattinoni na população de pacientes com insuficiência respiratória aguda hipoxêmica. Segundo este autor, apenas uma pequena parte do pulmão estaria disponível para a ventilação corrente, enquanto outra parte do parênquima pulmonar estaria indisponível à ventilação devido a colapso, edema alveolar e consolidações pneumônicas ${ }^{31}$. Baseado no princípio estabelecido por Gattinoni e colaboradores, Amato e colaboradores propuseram uma estratégia ventilatória com volumes correntes reduzidos para pacientes com insuficiência respiratória aguda, o que, em última instância, promoveu redução da mortalidade ${ }^{(32,33)}$. 
Mais recentemente, Terragni e colaboradores realizaram um estudo no qual observaram por meio de análise de tomografias computadorizadas em pausa inspiratória ao final da inspiração com volume corrente de $06 \mathrm{~mL} . \mathrm{kg}^{-1}$ do peso ideal e pressão de platô para $30 \mathrm{cmH}_{2} \mathrm{O}$, que havia hiperinsuflação do ápice pulmonar em $33 \%$ dos pacientes estudados, sugerindo que mesmo a estratégia protetora ainda pode não ser adequada em pacientes com comprometimento pulmonar extenso ${ }^{(34)}$.

\subsubsection{Contribuição da TC para avaliação pulmonar em pacientes submetidos à anestesia geral}

Alguns estudos relatam que o aparecimento de atelectasias pode

ocorrer já nos primeiros cinco minutos após anestesia geral ${ }^{35,36,37}$. A reabsorção de gás causada por uso de elevadas frações inspiradas de oxigênio, o uso de medicamentos que promovem relaxamento da musculatura, o deslocamento em direção cefálica do diafragma sob o peso das estruturas abdominais têm sido apontados como mecanismos responsáveis pelo colapso pulmonar observado nos períodos intra e pósoperatórios.

Os primeiros estudos, descrevendo de maneira quantitativa, foram realizados por Hedenstierna e colaboradores, que mostraram a dinâmica da formação das atelectasias durante o período intraoperatório, assim como o impacto do uso de frações elevadas de oxigênio ${ }^{38}$, do tempo cirúrgico ${ }^{39}$ e do uso de manobras de hiperinsuflação pulmonar sobre o colapso alveolar ${ }^{40}$. A eficiência da tomografia computadorizada torácica na avaliação de 
atelectasias permite detectar colapso pulmonar, mesmo que este não seja clinicamente significativo. Em um estudo realizado por Lundh e colaboradores, avaliando oxigenação sanguínea e tomografias de tórax no pós-operatório imediato em 20 pacientes submetidos a cirurgias abdominais de pequeno porte, os autores descreveram o aparecimento de áreas de atelectasias, sobretudo nas regiões posteriores e nas basais dos pulmões, apesar de não ter havido alterações na oxigenação ${ }^{41}$. A crítica a esse estudo, no entanto, deve-se ao fato de que todos os pacientes eram classificados de risco anestésico-cirúrgico baixo, de acordo com os critérios propostos pela Sociedade Americana de Anestesiologia (ASA - American Society Anestesiology), além de que as cirurgias foram de pequeno porte e com o tempo cirúrgico curto.

A tomografia computadorizada de tórax permite ainda avaliar o efeito de estratégias ventilatórias, como o uso de pressão positiva ao final da inspiração e manobras de hiperinsuflação pulmonar sobre a morfologia pulmonar em pacientes submetidos à ventilação mecânica. Em um estudo avaliando a relação entre atelectasias e oxigenação arterial, observou-se que, embora houvesse melhora das densidades pulmonares na tomografia computadorizada de tórax, $10 \mathrm{~cm} \mathrm{H}_{2} \mathrm{O}$ de PEEP não exerciam impactos adicionais sobre a oxigenação. Esse estudo evidenciou que o PEEP altera a relação de shunt pulmonar e atelectasias por distribuição do fluxo sanguíneo em direção a regiões atelectasiadas e shunt dependentes ${ }^{20}$. Rothen e colaboradores observaram, por meio de imagens de tomografia computadorizada da base pulmonar obtida a cada 1 segundo, que ocorria a 
reversão quase completa do colapso pulmonar em torno de 7 segundos, quando era aplicada uma pressão de $40 \mathrm{~cm} \mathrm{H2O}$ nas vias aéreas de pacientes submetidos à anestesia geral ${ }^{13}$.

As atelectasias são seis vezes mais frequentes após cirurgia cardíaca do que após cirurgias abdominais ${ }^{20}$. A parada da circulação e ventilação pulmonar durante a circulação extracorpórea também pode contribuir para a formação de atelectasias e, sobretudo, para o aparecimento de reação inflamatória do parênquima pulmonar ${ }^{33,35,36,37 .}$. 
MÉTodos

$\overline{=}$ 


\section{MÉTODOS}

\subsection{Pacientes}

Após a aprovação do Comitê de Ética do Hospital das Clínicas da Faculdade de Medicina da Universidade de São Paulo, pacientes com indicação de cirurgia para revascularização do miocárdio com CEC foram selecionados para participar do estudo. O projeto de pesquisa foi financiado a partir de recursos oriundos da FAPESP (Fundação de Amparo à Pesquisa do Estado de São Paulo), processo número 2005/0174-7.

O termo de consentimento pós-informado, livre e esclarecido (TCLE) foi obtido no dia anterior à cirurgia, após a apresentação e prestação de esclarecimentos sobre o estudo.

Os critérios de inclusão foram: 1) indicação de cirurgia de revascularização do miocárdio com circulação extracorpórea; 2) ausência de insuficiência cardíaca esquerda, definida como fração de ejeção do ventrículo esquerdo $<50 \%$ estimada por ecocardiografia ou ventriculografia. Os critérios de exclusão foram: 1) presença de doença pulmonar obstrutiva crônica; 2) insuficiência respiratória pré-operatória, caracterizada como necessidade de oxigênio suplementar pré-operatório; 3) congestão pulmonar pré-operatória; 4) índice de massa corpórea (IMC) maior que $30 \mathrm{~kg} / \mathrm{cm}^{2} ; 5$ ) necessidade de transfusão de mais que 03 unidades de qualquer hemocomponente; 6) insuficiência respiratória pós-operatória; e 7) instabilidade hemodinâmica, definida como necessidade de dobutamina em 
dose superior a $5 \mu \mathrm{g} \cdot \mathrm{kg}^{-1} \cdot \mathrm{min}$, uso de fármaco vasopressor em qualquer dose ou necessidade de assistência ventricular mecânica pré ou pósoperatória necessidade de sedação no pós-operatório por quaisquer motivos.

Um total de 40 pacientes foi selecionado e incluído no estudo desde o início do piloto do projeto, cuja coleta iniciou-se em novembro de 2005 (figura 01- painel superior).

Destes, 20 pacientes foram excluídos desde o início da coleta, em respeito aos critérios de inclusão ou exclusão. Os motivos que causaram a exclusão de 20 pacientes do projeto foram: dez pacientes permaneceram em ventilação mecânica por julgamento clínico, devido à necessidade de adequação hemodinâmica no primeiro dia pós-operatório, o que inviabilizou a realização da tomografia pós-operatória. Três pacientes cursaram com infarto agudo do miocárdio transoperatório e outros três pacientes evoluíram com instabilidade hemodinâmica já no intraoperatório, com necessidade de fármacos vasopressores em altas doses. Dois pacientes receberam mais de 03 unidades de hemocomponentes durante o intraoperatório. Um paciente recusou-se a realizar a tomografia pós-operatória e um paciente necessitou de ventilação não-invasiva com pressão positiva logo após a extubação (figura 01- painel inferior). 
Figura 1 - Total de pacientes selecionados

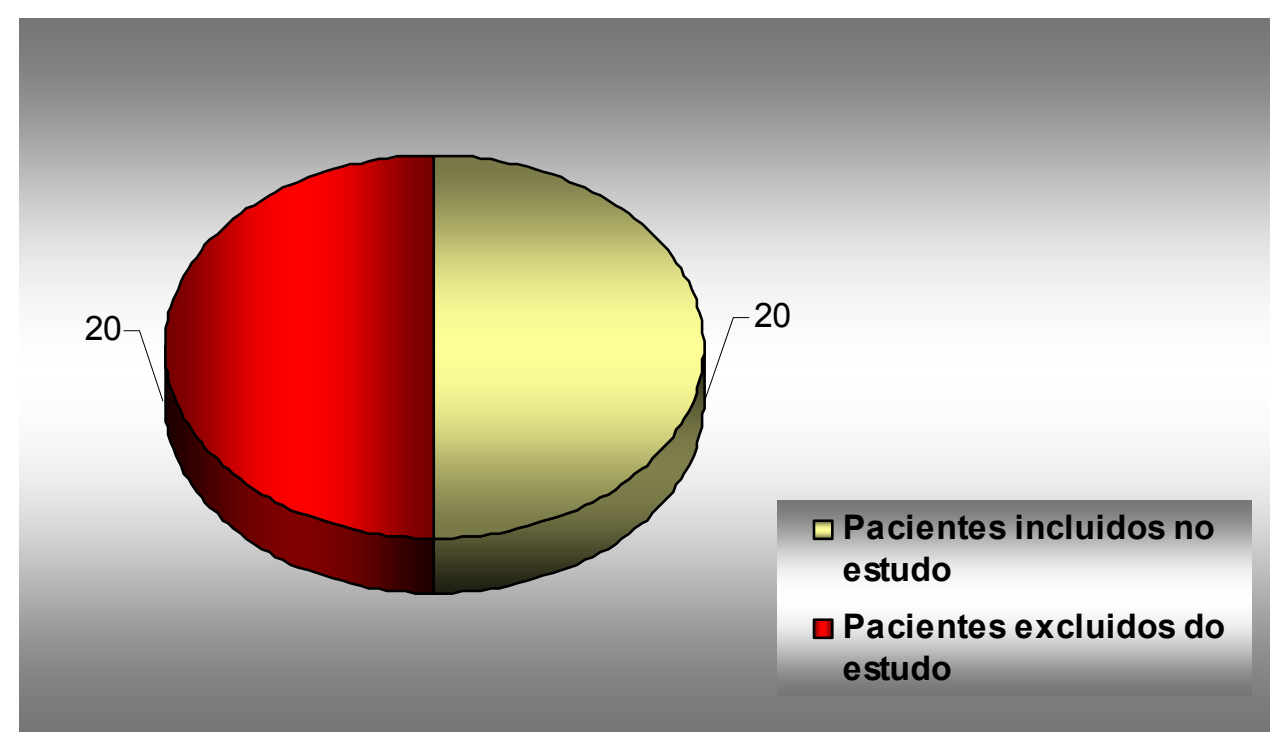

\section{Pacientes excluídos do estudo}

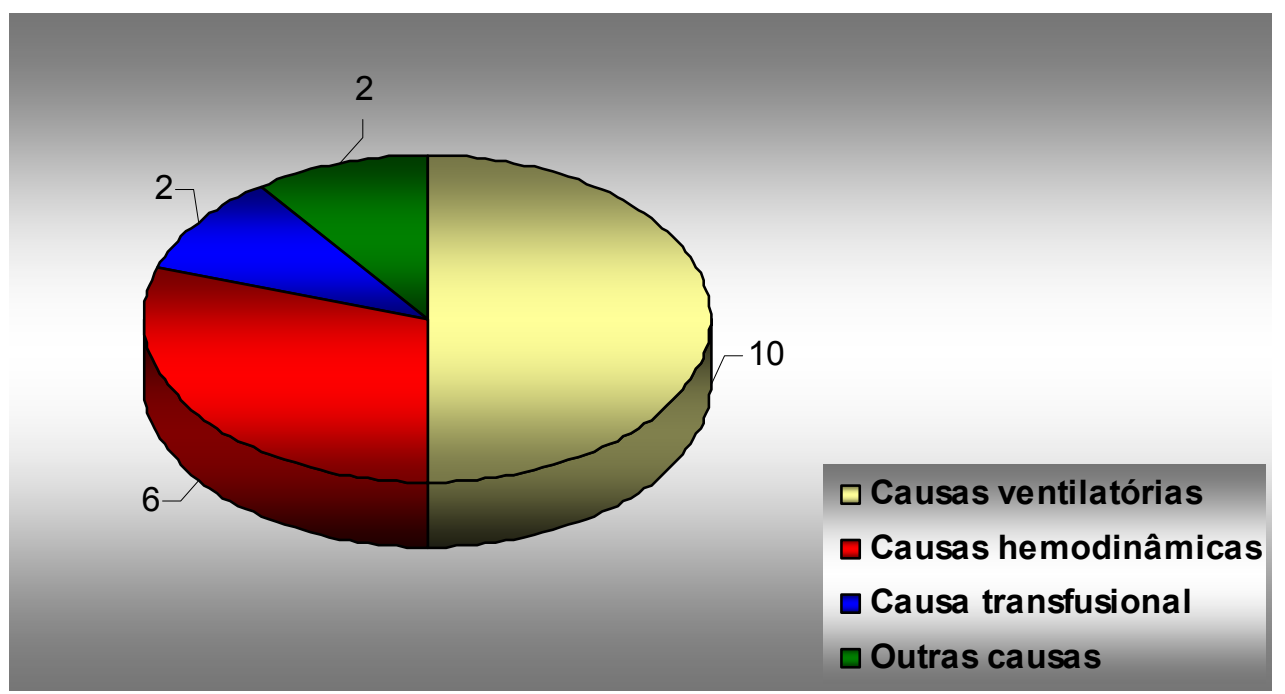

Obs: Após a execução do estudo, 02 (dois) pacientes do grupo de incluídos, saíram do protocolo, por falha na reconstrução perfeita das imagens tomográficas (artefatos). Desta forma, o total da amostra final foi reduzido para 18 pacientes. 


\subsection{Desenho de estudo}

Como pode ser observado na figura 02 (cronograma), segue o delineamento experimental nos diversos momentos do estudo:

\section{Figura 02}

\section{AdMISSÃO - DIA ANTERIOR À CIRURGIA}

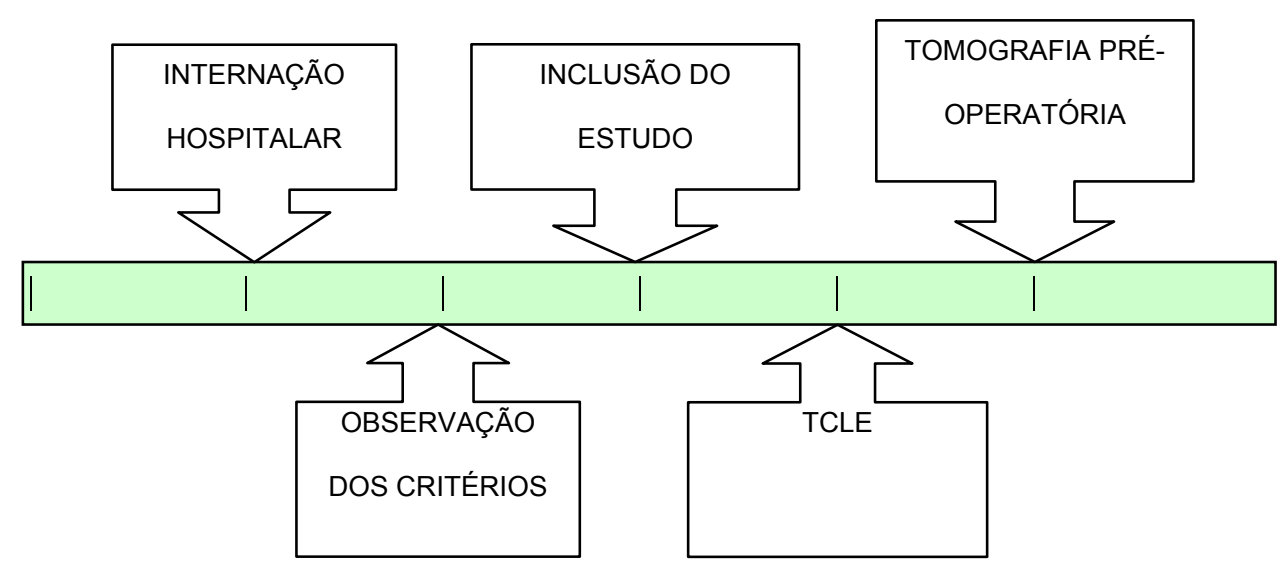

2. DIA DA CIRURGIA

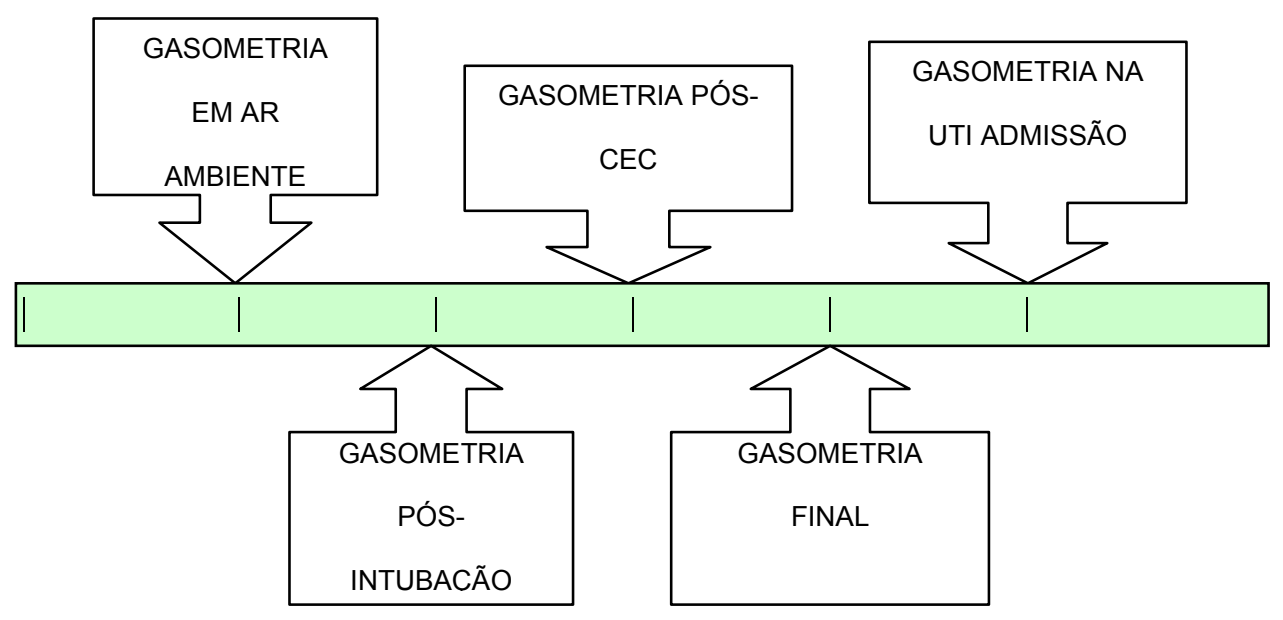




\section{UTI}

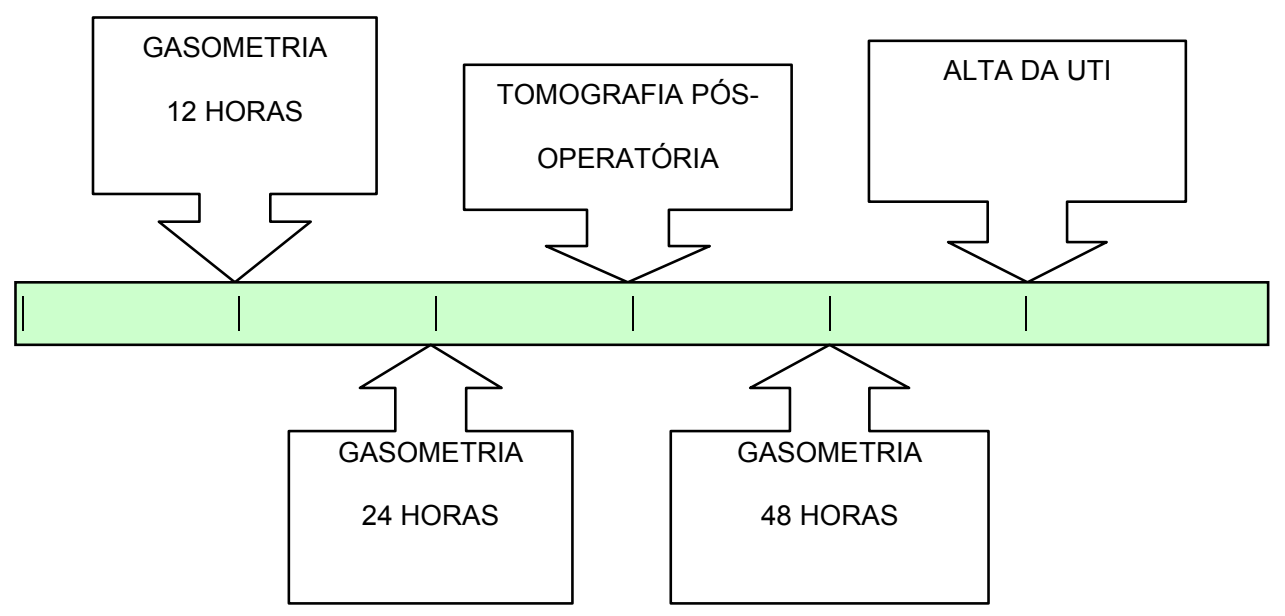

No dia anterior à cirurgia, após a explicação do estudo aos pacientes e obtenção do TCLE, os selecionados foram transportados por meio de cadeira de rodas para o setor de Radiologia do Instituto do Coração, para realização de TC helicoidal pré-operatória. As TC foram obtidas em ventilação espontânea, na condição de pausa expiratória, após uma expiração normal. Para alcançar esta exata condição respiratória, cada paciente foi treinado a realizar apneia após uma expiração normal durante 15 segundos necessários para obtenção das imagens da TC. Esse exercício foi repetido pelo menos 10 vezes após a assinatura do termo de consentimento. O mesmo exercício foi realizado mais duas vezes na sala de radiologia, antes da realização da TC. O objetivo desta manobra era obter as imagens de TC em condição respiratória próxima à capacidade residual funcional (CRF).

No primeiro dia pós-operatório, uma segunda tomografia computadorizada em torno de 24 horas após a admissão à UTI foi realizada, 
com os pacientes hemodinamicamente estáveis, extubados e sem necessidade de ventilação não-invasiva (VNI), de forma que todos os critérios de exclusão foram respeitados.

Os parâmetros hemodinâmicos durante o estudo foram obtidos de maneira padronizada, conforme descrito na literatura médica, usando cateter de artéria pulmonar e monitor hemodinâmico que permitiam a mensuração contínua do débito cardíaco. Estas medidas foram realizadas após a indução anestésica, após a saída de CEC e na UTI pós-operatória nos seguintes momentos: admissão, $12 \mathrm{~h}, 24 \mathrm{~h}$ de chegada à UTI. No momento 24 horas após a admissão à UTI, foi calculado o shunt intrapulmonar de maneira padrão.

Em cada paciente, uma gasometria arterial pré-operatória foi obtida no momento da admissão à sala de operações. Gasometrias arteriais e venosas foram colhidas após a intubação, após a saída de CEC e na UTI pós-operatória nos seguintes momentos: admissão, 12h, $24 \mathrm{~h}$ e $48 \mathrm{~h}$. As TC torácicas pós-operatórias foram obtidas no máximo até 24 horas após a cirurgia. Em cada momento, a fração inspirada de oxigênio foi registrada conforme ajuste do ventilador ou computada como $4 \%$ por litro de oxigênio acima de $21 \%$ (ar ambiente) nos pacientes em uso de cateter de oxigênio ${ }^{42}$.

\subsection{Técnica anestésica, monitorização e cuidados pós- operatórios}

No dia da cirurgia, cada paciente recebeu medicação préanestésica $0,2 \mathrm{mg} \cdot \mathrm{kg}^{-1}$ de midazolam por via oral até o máximo de $15 \mathrm{mg} 30$ minutos antes da admissão na sala de cirurgia. A monitorização 
hemodinâmica consistiu de eletrocardiograma contínuo (ECG) de 05 cabos (derivações DIl e V5), capnografia, oximetria de pulso, temperatura esofágica e pressão arterial invasiva. A anestesia foi induzida com fentanil $10 \mu \mathrm{g} \cdot \mathrm{kg}^{-1}$, etomidato $0,3 \mathrm{mg} \cdot \mathrm{kg}^{-1}$ e pancurônio 0,1 a $0,2 \mathrm{mg} \cdot \mathrm{kg}^{-1}$ e mantida com isoflurano inalatório a $1 \%$. Doses suplementares de midazolan $(0,3$ mg. $\left.\mathrm{Kg}^{-1}\right)$ e pancurônio $\left(0,08 \mathrm{mg} \cdot \mathrm{Kg}^{-1}\right)$ foram administradas imediatamente após o início da CEC, com a finalidade de manter a hipnose intraoperatória e evitar movimentos involuntários, respectivamente. Após a indução anestésica, um cateter de artéria pulmonar $\left(\mathrm{CCO} / \mathrm{SvO}_{2}, \mathrm{VIPTMTD}\right.$ cateter Edwards lifesciences, Irvine, CA, USA) foi inserido pela veia jugular interna direita. Para todos os pacientes, o volume corrente foi ajustado em $08 \mathrm{~mL} . \mathrm{kg}^{-}$ ${ }^{1}$ de acordo com o peso ideal ${ }^{43}$, e a frequência respiratória foi ajustada para manter a $\mathrm{PaCO}_{2}$ entre 30 e $35 \mathrm{mmHg}$. A relação inspiração/expiração foi de 1:2 do tempo total do ciclo respiratório e uma fração inspirada de oxigênio de 0,60 foi mantida durante todo o procedimento. Pressão positiva expiratória final (PEEP) de $05 \mathrm{~cm} / \mathrm{H}_{2} \mathrm{O}$ foi aplicada após intubação orotraqueal e mantida durante todo o procedimento.

Após a abertura do tórax e dissecção das estruturas vasculares, foi administrado heparina 500 Ul. $\mathrm{kg}^{-1}$ e inseridas cânulas cirúrgicas na aorta e nas veias cavas superior e inferior. A circulação extracorpórea foi realizada usando circuito de circulação extracorpórea e oxigenador de membrana (Braile, São José do Rio Preto, Brasil), preenchido com 1500 mL de solução de Ringer Lactato, manitol 1 g. $\mathrm{kg}^{-1}$ e heparina 10000 UI. Como rotina, a ventilação pulmonar foi interrompida imediatamente após o início da CEC, 
para facilitar a exposição cirúrgica das estruturas intratorácicas. Após o final da revascularização do miocárdio e imediatamente antes de reiniciar a ventilação mecânica, uma manobra de expansão pulmonar com $30 \mathrm{cmH}_{2} \mathrm{O}$ de pressão durante 20 segundos foi aplicada para reverter o colapso pulmonar relacionado à manipulação intratorácica, à desconexão ao circuito respiratório e à pausa ventilatória. Fármacos vasoativos e inotrópicos foram infundidos, quando necessário, ao final da CEC.

A hidratação no intraoperatório fora uniformizada de forma a repor o jejum $\left(02 \mathrm{ml} . \mathrm{Kg} \cdot \mathrm{h}^{-1}\right)$, as perdas insensíveis $\left(08 \mathrm{ml} . \mathrm{Kg} \cdot \mathrm{h}^{-1}\right)^{44}$, diurese e perdas eventuais do intraoperatório, baseada em avaliação de aspiradores e peso de compressas. As pressões de enchimento do cateter de artéria pulmonar e lactato séricos também foram avaliadas de forma que foram objetivados os valores referência de normalidade ${ }^{45}$.

Após a cirurgia, os pacientes foram transportados para a UTI de pós-operatório. Após a reconexão ao ventilador mecânico na UTI, uma segunda manobra de expansão pulmonar com $30 \mathrm{cmH}_{2} \mathrm{O}$ de pressão durante 20 segundos foi realizada e mantido PEEP de $5 \mathrm{cmH}_{2} \mathrm{O}$. Os pacientes permaneceram sem sedação, e, tão logo conscientes, foram extubados, desde que apresentassem os seguintes critérios: 1) estabilidade hemodinâmica (definida como pressão arterial média maior ou igual a 70 mmHg, saturação venosa central maior que $70 \%$, frequência cardíaca menor que 120 bpm, sem sinais de isquemia miocárdica); 2) adequada função pulmonar $\left(\mathrm{PaO}_{2}>80 \mathrm{mmHg}\right.$ e $\mathrm{PaCO}_{2}<45 \mathrm{mmHg}$ e $\mathrm{FiO}_{2}<0,4$, frequência respiratória espontânea menor que $30 \mathrm{ipm} \cdot \mathrm{min}^{-1}$, volume corrente maior que 
$05 \mathrm{ml} . \mathrm{kg}^{-1}$ ); 3) força muscular preservada (ventilação espontânea, habilidade de sustentar a cabeça elevada por mais de 5 segundos); 4) consciência (capacidade de obedecer comandos verbais simples) e 5) ausência de complicações cirúrgicas.

Após a extubação, os pacientes receberam oxigênio sob cateter nasal, de maneira que a oximetria de pulso fosse superior a $92 \%$. Não foi aplicado suporte ventilatório não-invasivo após a extubação

Analgesia pós-operatória foi instituída tão logo os pacientes referiam dor ou imediatamente após a extubação. Os fármacos usados para a analgesia foram tramadol $400 \mathrm{mg} \cdot \mathrm{dia}^{-1}$ e dipirona sódica 08 gramas.dia $^{-1}$. Resgates com 02 mg de morfina foram usados nos casos de dor refratária a terapia adotada. Apenas 04 pacientes necessitaram de doses suplementares de morfina, num total estimado em 3,8mg/dia. Nenhum paciente necessitou de instalação de bomba de ACP (Analgesia Controlada pelo Paciente). No momento da realização das tomografias pós-operatórias, nenhum paciente referia dor na inspiração profunda.

\subsection{Protocolo de estudo por tomografia computadorizada}

\subsubsection{Aquisição das tomografias computadorizadas.}

Cada paciente foi transportado ao departamento de radiologia por um médico participante do estudo. No primeiro dia pós-operatório, os pacientes foram monitorizados durante o transporte e na sala de tomografia computadorizada usando ECG, oximetria de pulso e pressão arterial invasiva 
com o monitor de transporte Philips M3 (Philips, Eindhoven, The Netherlands).

As aquisições das tomografias volumétricas helicoidais do tórax foram realizadas por um tomógrafo Toshiba de 16 canais. As exposições foram feitas a 120 kV e 250 mAs. O tempo de rotação foi 1 segundo, colimação $10 \mathrm{~mm}$. As imagens contínuas axiais foram reconstruídas usando o algoritmo de reconstrução do equipamento de tomografia computadorizada, com espessura de $10 \mathrm{~mm}$, a partir dos dados volumétricos. As imagens foram registradas em uma janela para parênquima pulmonar (largura da janela $=1400 \mathrm{UH}$ e centro da janela $=-400 \mathrm{UH}$ ).

As imagens reconstruídas foram gravadas no sistema de armazenamento de imagem do hospital e recuperadas em um computador pessoal compatível com a plataforma IBM-PC, para posterior análise. A análise das imagens reconstruídas foi realizada em um computador, usando um programa concebido para medidas quantitativas a partir de imagens de tomografia computadorizada (Osiris 4.19, Hospital Universitário de Geneva, Geneva, Suíça).

\subsubsection{Avaliação do volume pulmonar de gás, tecido e total.}

O volume pulmonar total foi computado como o número total de vóxels (unidade elementar volumétrica da tomografia computadorizada), cujas dimensões são conhecidas. Os volumes de gás e tecido foram medidos conforme o método descrito por Puybasset et al ${ }^{46,47,48}$, baseado na correlação linear entre o coeficiente de atenuação de um determinado material aos raios $X$ (coeficiente CT) e a densidade física ${ }^{49}$. O coeficiente 
CT caracterizando cada vóxel é definido como o coeficiente de atenuação dos raios $\mathrm{X}$ pelo material estudado menos o coeficiente de atenuação dos raios $\mathrm{X}$ pela água, dividido pelo coeficiente de atenuação dos raios $\mathrm{X}$ pela água, expresso em unidades Hounsfield (UH). Por convenção, o coeficiente CT da água é 0 UH e o coeficiente CT de gás é -1000 UH. Um vóxel pulmonar caracterizado por uma densidade radiológica de -500 UH é constituído de $50 \%$ de gás e $50 \%$ de tecido. Um vóxel caracterizado por uma densidade radiológica de -200 UH é constituído de $20 \%$ de gás e $80 \%$ de tecido. Usando esta análise, é possível computar o volume de gás e tecido presente no pulmão.

Inicialmente, os vóxels com densidade radiológica entre -1200 UH e +200 UH foram distribuídos em 1200 compartimentos com intervalo de 1 UH. Para cada compartimento com um número de vóxels conhecido, o volume total, o volume de gás e o volume de tecido foram computados usando as fórmulas seguintes:

(1) Volume do vóxel = (área do píxel) $x$ espessura da seção, sendo que a área do píxel é fornecida para cada estudo tomográfico;

(2) Volume total do compartimento = número de vóxels $x$ volume do vóxel para cada faixa de coeficiente CT;

(3) Volume de gás $=(-$ coeficiente CT / 1000) $\times$ volume total do compartimento, se o compartimento considerado tem densidade radiológica entre -1000 UH e 0 UH, (3') volume de gás $=0$, se o compartimento considerado tem coeficiente CT maior 0 UH e (3") volume de gás = volume total do compartimento, se o tem coeficiente CT menor que $-1000 \mathrm{UH}$; 
(4) Volume de tecido $=(1000+$ coeficiente CT / 1000 $) \times$ volume total do compartimento, se o compartimento considerado tem densidade radiológica entre $-1000 \mathrm{UH}$ e $0 \mathrm{UH},\left(4^{\prime}\right)$ volume de tecido = número de vóxels x volume do vóxel, se o compartimento considerado tem coeficiente CT maior 0 UH e (4") volume de tecido $=0$, se o compartimento considerado tem coeficiente CT menor que $-1000 \mathrm{UH}$;

(5) Peso do parênquima pulmonar = volume de tecido, se o compartimento considerado tem coeficiente CT menor que $0 \mathrm{UH}$ e (5') peso do parênquima pulmonar $=(1000+$ coeficiente CT $/ 1000) \times$ volume total do compartimento, se o compartimento considerado tem coeficiente CT maior que $0 \mathrm{UH}$.

Num segundo tempo, o volume total de gás, de tecido e o peso do parênquima pulmonar de uma determinada região de interesse foram computados somando os volumes e o peso respectivos dos compartimentos analisados nesta região. A capacidade residual funcional, o volume de tecido pulmonar total e o volume pulmonar total foram computados, somando os volumes parciais de gás, tecido e o volume total de todos os compartimentos contidos no pulmão.

O volume de tecido mensurado a partir de tomografia computadorizada representa a soma dos volumes do parênquima pulmonar, sangue e seus elementos figurados e água extravascular pulmonar. 


\subsubsection{Análise da aeração pulmonar de acordo com a distribuição dos coeficientes CT.}

Paralelamente à determinação dos volumes de gás e tecido do pulmão, a tomografia computadorizada permite estudar o parênquima pulmonar em função do seu grau de aeração. Este tipo de análise permite classificar o parênquima pulmonar de acordo com seu coeficiente.

1) Região com parênquima pulmonar hiperinsuflado: coeficientes CT compreendidos entre -1000 UH e -900 UH.

2) Região com parênquima pulmonar normalmente aerado: coeficientes CT compreendidos entre $-900 \mathrm{UH}$ e -500 UH.

3) Região com parênquima pulmonar mal aerado: coeficientes CT compreendidos entre $-500 \mathrm{UH}$ e $-100 \mathrm{UH}$.

4) Região com parênquima pulmonar não aerado (atelectasiado): coeficientes CT compreendidos entre $-100 \mathrm{UH}$ e $+100 \mathrm{UH}$.

Estes limites indicam que a "região hiperinsuflada" contém mais de $90 \%$ de gás; a "região normalmente aerada" entre $50 \%$ e $90 \%$ de gás; a “região mal aerada" entre $50 \%$ e $10 \%$ e a "região não aerada" menos de $10 \%$ de gás. Contudo, para evitar erros de análise das regiões pulmonares quanto ao grau de aeração, foi considerado o peso do parênquima pulmonar, ao invés do volume total da região. 


\subsection{Análise estatística}

\subsubsection{Cálculo amostral}

Uma amostra de pelo menos 17 pacientes era necessária para encontrar o aumento de volume de colapso pulmonar pós-operatório de 15\%, comparado com as TC pré-operatórias, considerando um desvio padrão de $10 \%$ pelo teste pareado de T Student, com um valor $\mathrm{p}$ menor que 0,05 e um poder de estudo de 0,9. O tamanho da amostra foi calculado

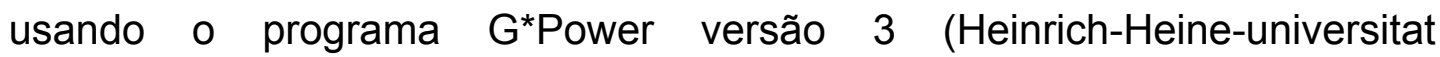
Dusseldorf, Dusseldorf, Germany).

As análises estatísticas foram realizadas usando SPSS 15.0 (SSPS Inc., Chicago, IL,EUA) e SIGMASTAT (Systat Software Inc, San Jose, CA, EUA). A distribuição normal dos dados passou pelo teste de Kolmogorov-Smirnov. O comportamento temporal dos dados hemodinâmicos e outras variáveis fisiológicas foram analisados ao longo do tempo, usando análise de variância de uma via para repetidas medidas, seguidos pelo teste de Student-Neumann-Keuls, quando necessário. Dados volumétricos e de peso pulmonar foram obtidos a partir das análises tomográficas, sendo comparados por meio de teste $\mathrm{T}$ de Student pareado. As correlações entre os dados obtidos a partir da análise de tomografias computadorizadas e dados fisiológicos foram realizadas por meio de análise de regressão linear ou teste de correlação ordinal de Spearman. Todos os dados estão apresentados como média $\pm S D$, valores medianos (min-máx) ou conforme descrito quando apresentado de outro modo. O nível de significância foi fixado em $5 \%$. 


\section{RESULTADOS}

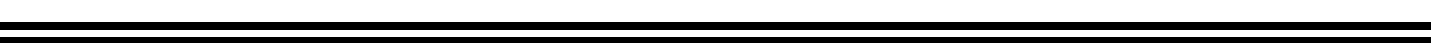




\section{Resultados}

Dos 20 pacientes selecionados para o estudo, que assinaram o termo de consentimento e completaram a coleta de dados, 02 pacientes foram excluídos, porque a tomografia computadorizada de tórax pósoperatória apresentava muitos artefatos de movimento que impediram a adequada análise das imagens da TC pós-operatório (esses pacientes não conseguiram manter apneia).

As características demográficas dos 18 pacientes estudados estão apresentadas na Tabela I. A média de idade foi $63 \pm 9$ anos, com um peso de $77 \pm 12 \mathrm{~kg}$. A média de duração da cirurgia foi $420 \pm 47$ minutos e a média de duração da CEC foi $90 \pm 20$ minutos Tabela II. Os pacientes receberam $4227 \pm 1039 \mathrm{ml}$ de solução cristaloide, $909 \pm 202 \mathrm{ml}$ de solução de hidroxietilamido a $6 \%$ durante o período intraoperatório e o volume de diurese intraoperatório foi $1016 \pm 562 \mathrm{ml}$. Do total de líquido infundido durante a cirurgia, $2433 \pm 1806 \mathrm{ml}$ foram administrados durante a CEC. O volume de sangramento estimado foi $345 \pm 248 \mathrm{ml}$, e nove pacientes receberam uma unidade de concentrado de hemácias. A dobutamina foi infundida em 15 pacientes, para facilitar o desmame da CEC, sendo empregada em dose média $8 \pm 3 \mu \mathrm{g} \cdot \mathrm{kg}^{-1} \cdot \mathrm{min}$. Todos os pacientes evoluíram com estabilidade hemodinâmica no pós-operatório imediato, permitindo a suspensão dos fármacos vasoativos nas primeiras 24 horas. O tempo médio 
para extubação foi $435 \pm 182$ minutos. Infusão de fluidos no pós-operatório foi $1550 \pm 257 \mathrm{ml}$.

Tabela I - Dados demográficos e clínicos dos pacientes estudados

\begin{tabular}{|c|c|c|c|c|c|c|c|c|c|c|}
\hline Paciente & Idade (anos) & Peso (kg) & Altura (cm) & FEVE (\%) & $\mathrm{Hb}\left(\mathrm{g} . \mathrm{dL}^{-1}\right)$ & $\operatorname{Cr}\left(\mathrm{g} \cdot \mathrm{dL}^{-1}\right)$ & Reoperação & DPOC & Hipertensão & DM \\
\hline DRB & 48 & 66 & 162 & 62 & 14.7 & 0.9 & $\mathrm{~N}$ & $\mathbf{N}$ & $\mathrm{N}$ & $N$ \\
\hline MMF & 51 & 78 & 175 & 55 & 13.7 & 0.8 & $\mathrm{~N}$ & $\mathbf{N}$ & S & $S$ \\
\hline LFG & 62 & 112 & 185 & 50 & 12.9 & 1.3 & $N$ & N & s & s \\
\hline JA & 77 & 70 & 170 & 73 & 14 & 1.1 & $\mathrm{~N}$ & $\mathbf{N}$ & $\mathbf{s}$ & $N$ \\
\hline RFFS & 65 & 90 & 171 & 70 & 13.7 & 0.9 & $N$ & $\mathbf{N}$ & $s$ & $\mathrm{~s}$ \\
\hline OP & 58 & 72 & 170 & 57 & 14.5 & 1 & $N$ & $\mathbf{N}$ & s & $s$ \\
\hline ACPF & 62 & 81 & 170 & 50 & 14.6 & 0.8 & $N$ & $\mathbf{N}$ & $s$ & s \\
\hline ORC & 74 & 74 & 154 & 55 & 13 & 1.1 & $s$ & $\mathbf{N}$ & $s$ & $N$ \\
\hline AO & 74 & 89 & 180 & 50 & 13.4 & 1.2 & $\mathrm{~N}$ & $\mathbf{N}$ & $s$ & $s$ \\
\hline MNM & 62 & 75 & 165 & 78 & 13.5 & 0.9 & $\mathrm{~N}$ & $\mathbf{N}$ & $\mathrm{N}$ & $s$ \\
\hline DPC & 72 & 62 & 164 & 50 & 15.8 & 0.9 & $N$ & N & $s$ & $N$ \\
\hline MC & 62 & 83 & 165 & 55 & 11.2 & 1.3 & $s$ & $\mathbf{N}$ & s & $N$ \\
\hline A.JF & 71 & 72 & 172 & 60 & 14.9 & 1 & $N$ & N & s & $N$ \\
\hline JBM & 52 & 74 & 172 & 69 & 16.2 & 1.1 & $N$ & N & s & $N$ \\
\hline $\mathrm{JC}$ & 67 & 63 & 158 & 58 & 13.6 & 1 & $N$ & $\mathbf{N}$ & s & $N$ \\
\hline IS & 56 & 63 & 153 & 57 & 12.8 & 1.3 & $\mathbf{N}$ & $\mathbf{N}$ & $\mathrm{N}$ & $N$ \\
\hline AC & 73 & 70 & 167 & 70 & 15.9 & 1.2 & $\mathrm{~N}$ & $\mathbf{N}$ & $s$ & $S$ \\
\hline GAT & 50 & 84 & 160 & 61 & 13.5 & 1.3 & $\mathrm{~N}$ & $\mathbf{N}$ & $s$ & $\mathrm{~N}$ \\
\hline
\end{tabular}

Legenda: FEVE - fração de ejeção do ventrículo esquerdo; $\mathrm{Hb}$ hemoglobina; $\mathrm{Cr}$ - creatinina; DPOC - doença pulmonar obstrutiva crônica; $\mathrm{DM}$ - diabetes melitus; $\mathrm{N}$ - não e $\mathrm{S}$ - sim. 
Tabela II - Dados relacionados ao tempo de CEC, duração da cirurgia, volume total de diurese $e$ volume infundido no intraoperatório

\begin{tabular}{lccccc}
\hline \hline & $\begin{array}{c}\text { Duração da } \\
\text { CEC } \\
\text { (minutos) }\end{array}$ & $\begin{array}{c}\text { Tempo de } \\
\text { cirurgia } \\
\text { (minutos) }\end{array}$ & $\begin{array}{c}\text { Diurese } \\
\text { intraoperatória } \\
\text { (ml) }\end{array}$ & $\begin{array}{c}\text { Volume (ml) } \\
\text { infundido } \\
\text { intraoperatório } \\
\text { (cristaloides) }\end{array}$ & $\begin{array}{c}\text { Volume (ml) } \\
\text { infundido } \\
\text { intraoperatório } \\
\text { (coloides) }\end{array}$ \\
\hline \hline DRB & 110 & 450 & 1500 & 3000 & 1000 \\
MMF & 96 & 390 & 800 & 4500 & 0 \\
LFG & 100 & 360 & 500 & 3000 & 1000 \\
JÁ & 125 & 370 & 500 & 4500 & 1000 \\
RFFS & 100 & 330 & 1500 & 2000 & 1000 \\
OP & 142 & 350 & 1500 & 4500 & 1000 \\
ACPF & 82 & 315 & 1400 & 4000 & 0 \\
ORC & 80 & 350 & 2000 & 4700 & 1000 \\
AO & 75 & 330 & 1000 & 3000 & 0 \\
MNM & 45 & 375 & 1400 & 5600 & 1000 \\
DPC & 110 & 360 & 450 & 6000 & 0 \\
MC & 90 & 415 & 2000 & 5500 & 0 \\
AJF & 50 & 325 & 300 & 4000 & 1000 \\
JBM & 95 & 318 & 800 & 4500 & 0 \\
JC & 93 & 420 & 1000 & 4000 & 0 \\
IS & 78 & 360 & 1200 & 4500 & 0 \\
AC & 100 & 330 & 400 & 4600 & 0 \\
GAT & 98 & 370 & 450 & 5000 & 0 \\
\hline \hline
\end{tabular}

Nenhum dos 18 pacientes evoluiu com falha de extubação ou necessidade de ventilação não-invasiva no pós-operatório. Todos os pacientes receberam alta da UTI sem complicações, com tempo de permanência que variou de 38 a 48 horas. 


\subsection{Dados hemodinâmicos e trocas gasosas durante o período de estudo}

A Tabela III mostra o comportamento temporal dos dados hemodinâmicos, desde a indução da anestesia até 24 horas após a admissão a UTI, e a Tabela IV os dados respiratórios desde a indução da anestesia até 48 horas do pós-operatório.

Como pode ser observado na Tabela III, durante todo o período de observação, o índice cardíaco permaneceu acima de $2,5 \mathrm{I} \cdot \mathrm{min}^{-1} \cdot \mathrm{m}^{2}$. A pressão de artéria pulmonar atingiu seu valor máximo após a CEC $(27 \pm 7$ $\mathrm{mmHg}$ ), retornando aos valores normais, contudo sem alterações estatisticamente significativas. A média da pressão de oclusão da artéria pulmonar permaneceu abaixo de $18 \mathrm{mmHg}$ durante todo o estudo, apesar da infusão de fluidos realizadas no perioperatório. Houve aumento significativo da frequência cardíaca e redução nos valores do índice de resistência vascular sistêmica após a saída de CEC, que persistiram até o final do período de estudo. Não foram observadas variações significativas na pressão venosa central, no índice de resistência vascular pulmonar e no índice sistólico. 
Tabela III - Dados hemodinâmicos durante a cirurgia e nas primeiras 24 horas pós-operatórias

\begin{tabular}{|c|c|c|c|c|c|c|}
\hline & Após indução & Após CEC & Admissão UTI & UTI $12 \mathrm{~h}$ & UTI 24 h & valor $\mathrm{p}$ \\
\hline FC (BPM) & $69 \pm 20$ & $98 \pm 17^{\star}$ & $98 \pm 20^{\star}$ & $95 \pm 16^{\star}$ & $98 \pm 15^{\star}$ & $<0.001$ \\
\hline PAM (mmHg) & $74 \pm 9$ & $66 \pm 8^{*}$ & $84 \pm 15$ & $76 \pm 9$ & $81 \pm 9$ & $<0.001$ \\
\hline PAPm (mmHg) & $25 \pm 6$ & $27 \pm 7$ & $25 \pm 7$ & $23 \pm 7$ & $23 \pm 6$ & NS \\
\hline PVC (mmHg) & $14 \pm 6$ & $16 \pm 6$ & $14 \pm 5$ & $11 \pm 4$ & $12 \pm 4$ & NS \\
\hline POAP (mmHg) & $16 \pm 4$ & $17 \pm 5$ & $15 \pm 5$ & $14 \pm 5$ & $15 \pm 4$ & NS \\
\hline IC $\left(\right.$ L. $\left.\min ^{-1} \cdot \mathrm{m}^{2}\right)$ & $2.5 \pm 0.8$ & $3.9 \pm 1.4^{*}$ & $3.2 \pm 1.1^{*}$ & $3.3 \pm 0.9^{*}$ & $3.9 \pm 0.7^{*}$ & 0.003 \\
\hline IS (mL.m.2) & $38 \pm 12$ & $40 \pm 13$ & $34 \pm 13$ & $35 \pm 11$ & $41 \pm 10$ & NS \\
\hline IRVS (din.s. $\left.\mathrm{cm}^{-5} \cdot \mathrm{m}^{2}\right)$ & $2090 \pm 667$ & $1255 \pm 627^{*}$ & $1944 \pm 676$ & $1707 \pm 442^{*}$ & $1499 \pm 323^{*}$ & $<0.001$ \\
\hline IRVP (din.s. $\mathrm{cm}^{-5} \cdot \mathrm{m}^{2}$ ) & $312 \pm 220$ & $225 \pm 158$ & $239 \pm 129$ & $241 \pm 124$ & $168 \pm 93$ & NS \\
\hline
\end{tabular}

Legenda: FC - frequência cardíaca; PAM - pressão arterial média; PAPm pressão de artéria pulmonar média; PVC- pressão venosa central; POAPpressão de oclusão de artéria pulmonar; IC- índice cardíaco; IS- índice sistólico; IRVS- índice de resistência vascular sistêmica; IRVP- índice de resistência vascular pulmonar. Os dados estão expressos como média \pm desvio padrão. As variáveis foram analisadas pela análise de variância de uma via para repetidas medidas. $\left(^{*}\right)$ significa $p<0.05$, quando comparado com o momento após indução.

Todos os pacientes atingiram a estabilidade hemodinâmica no pós-operatório imediato, permitindo o desmame de fármacos vasoativos dentro das primeiras 24 horas.

Foi observada uma redução da saturação venosa mista para valores abaixo de $70 \%$ após 12 horas de admissão a UTI (Tabela IV), porém sem evidências clínicas e hemodinâmicas de disfunção miocárdica. O lactato arterial atingiu seus valores mais altos após o desmame da CEC, retornando 
aos níveis normais dentro das primeiras 12 horas de pós-operatório. Nenhuma alteração significativa foi observada quanto à diferença venoarterial da pressão parcial de $\mathrm{CO}_{2}$ e ao excesso de bases.

Tabela IV - Perfil metabólico durante a cirurgia e nas primeiras 48 horas pós-operatórias

\begin{tabular}{|c|c|c|c|c|c|c|c|c|}
\hline & Pré-indução & Após indução & Após CEC & Admissão UTI & UTI $12 \mathrm{~h}$ & UTI $24 \mathrm{~h}$ & UTI $48 \mathrm{~h}$ & valor $p$ \\
\hline $\mathrm{PaCO}_{2}(\mathrm{mmHg})$ & $38 \pm 5$ & $35 \pm 5$ & $38 \pm 5$ & $40 \pm 7$ & $35 \pm 4$ & $37 \pm 4$ & $38 \pm 5$ & NS \\
\hline $\mathrm{pH}$ Arterial & $7.44 \pm 0.05$ & $7.43 \pm 0.05$ & $7.33 \pm 0.08^{*}$ & $7.33 \pm 0.07^{\star}$ & $7.38 \pm 0.04$ & $7.4 \pm 0.05$ & $7.39 \pm 0.05$ & 0.001 \\
\hline Excesso de bases (mEq.L & $0.1 \pm 1.4$ & $0 \pm 1.6$ & $-4.5 \pm 3.3^{*}$ & $-4.2 \pm 4.05^{\star}$ & $-3 \pm 2^{*}$ & $-0.9 \pm 2.4$ & $-0.7 \pm 2.1$ & 0.003 \\
\hline Lactato Arterial $\left(\mathrm{mg} \cdot \mathrm{dL}^{-1}\right)$ & $13 \pm 3$ & $14 \pm 3$ & $36 \pm 20^{*}$ & $35 \pm 26^{*}$ & $19 \pm 7$ & $16 \pm 6$ & $14 \pm 6$ & 0.004 \\
\hline $\mathrm{PvCO}_{2}-\mathrm{PaCO}_{2}(\mathrm{mmHg})$ & - & $5 \pm 2$ & $3 \pm 4$ & $4 \pm 5$ & $4 \pm 4$ & $2 \pm 3$ & $5 \pm 4$ & NS \\
\hline $\mathrm{SvO}_{2}(\%)$ & - & $80 \pm 6$ & $78 \pm 10$ & $74 \pm 11$ & $65 \pm 9 !$ & $69 \pm 7 !$ & $65 \pm 9 !$ & 0.004 \\
\hline
\end{tabular}

Legenda: PvCO2-PaCO2 - diferença venoarterial da pressão parcial de $\mathrm{CO}_{2}$. Os dados estão expressos como média \pm desvio padrão. As variáveis foram analisadas pela análise de variância de uma via para repetidas medidas. $\left({ }^{*}\right)$ significa $p<0.05$, quando comparado com o momento préindução. (!) significa $p<0.05$, quando comparado com o momento após indução.

Quando analisado o comportamento da oxigenação arterial, como apresentado na Figura 03, foi observado que a relação $\mathrm{PaO}_{2} / \mathrm{FiO}_{2}$ diminuiu de forma significativa após a indução da anestesia, atingindo o seu nadir após a saída de CEC. Uma melhora significativa da relação $\mathrm{PaO}_{2} / \mathrm{FiO}_{2}$ foi observada após 12 horas da admissão a UTI, em comparação com os valores pós-CEC e se manteve até o final do estudo. Contudo, os valores da relação $\mathrm{PaO}_{2} / \mathrm{FiO}_{2}$ computados nas 24 e 48 horas de pós-operatório foram 
$26,1 \%$ e $17,7 \%$ menores que os valores de relação $\mathrm{PaO}_{2} / \mathrm{FiO}_{2}$ observados antes da indução anestésica $(p<0,05)$ respectivamente.

O shunt intrapulmonar médio computado nas primeiras 24 horas pós-operatórias foi $13 \pm 5 \%$. Foi encontrada uma fraca correlação negativa entre shunt intrapulmonar e relação $\mathrm{PaO}_{2} / \mathrm{FiO}_{2}(r=-0,47, p=0,04)$ obtida nas primeiras 24 horas. Nenhuma alteração foi observada na pressão parcial arterial de dióxido de carbono durante todo o estudo, como pode ser observado na Tabela IV. 


\section{FIGURA 3}

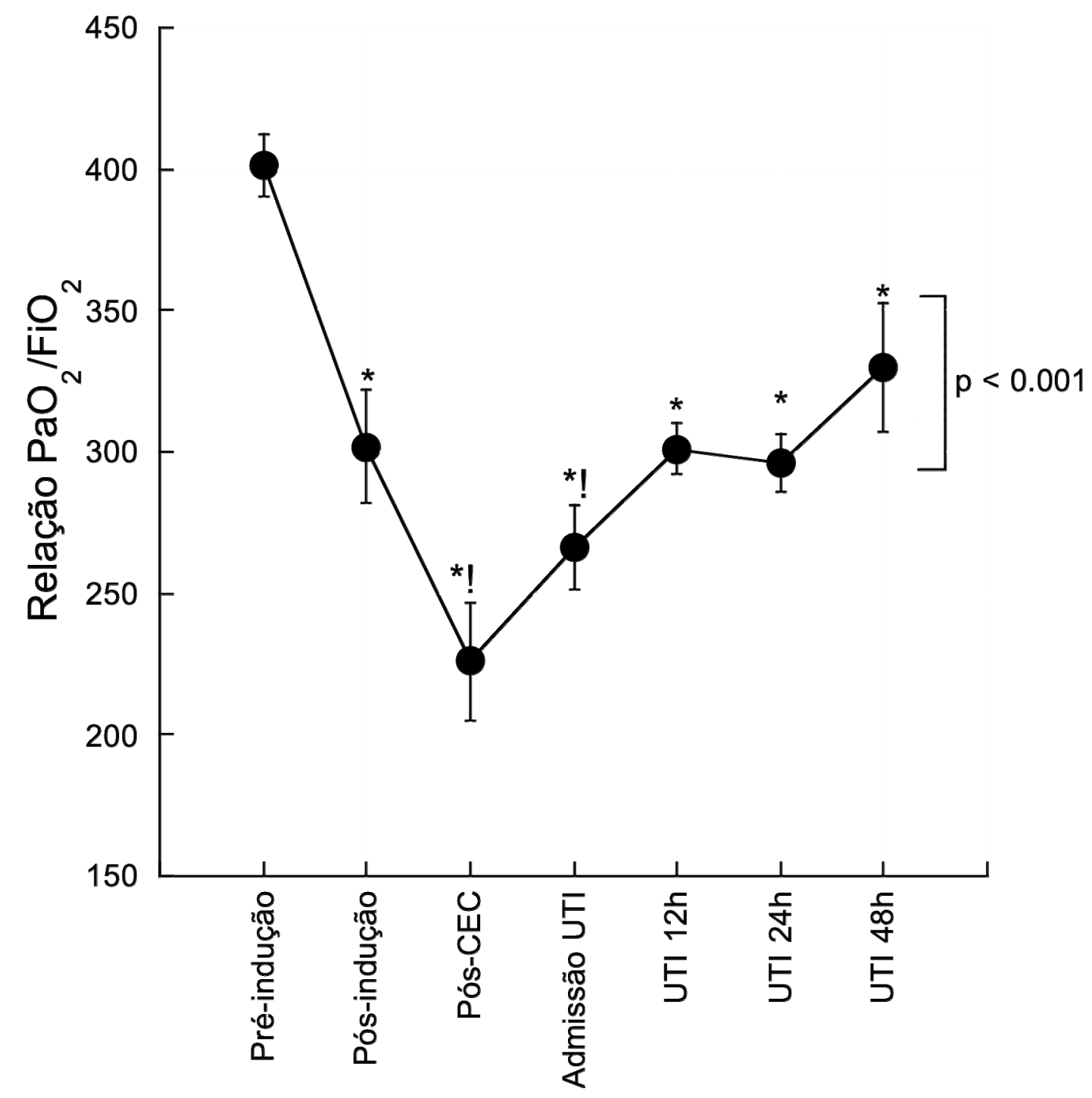

Figura 3 - Comportamento da relação $\mathrm{PaO}_{2} / \mathrm{FiO}_{2}$ durante o estudo. Os dados estão expressos como média \pm erro padrão. As variáveis foram analisadas pela análise de variância de uma via para repetidas medidas. $\left({ }^{*}\right)$ significa $p<0.05$, quando comparado com o momento pré-indução. (!) significa $p<0.05$, quando comparado com o momento após indução. 
5.2. Avaliação pulmonar pré e pós-operatória por meio de tomografia computadorizada

Figura 4: Imagens tomográficas representativas obtidas no ápice pulmonar (coluna da esquerda), no hilo pulmonar (coluna do meio) e próximo ao diafragma (coluna da direita) obtidas no pré-operatório (painel superior) e no primeiro dia pós-operatório (painel inferior). Observa-se a formação de imagens compatíveis com atelectasias nas regiões dependentes da TC do pós-operatório. Flechas mostram as áreas de colapsos pulmonares.

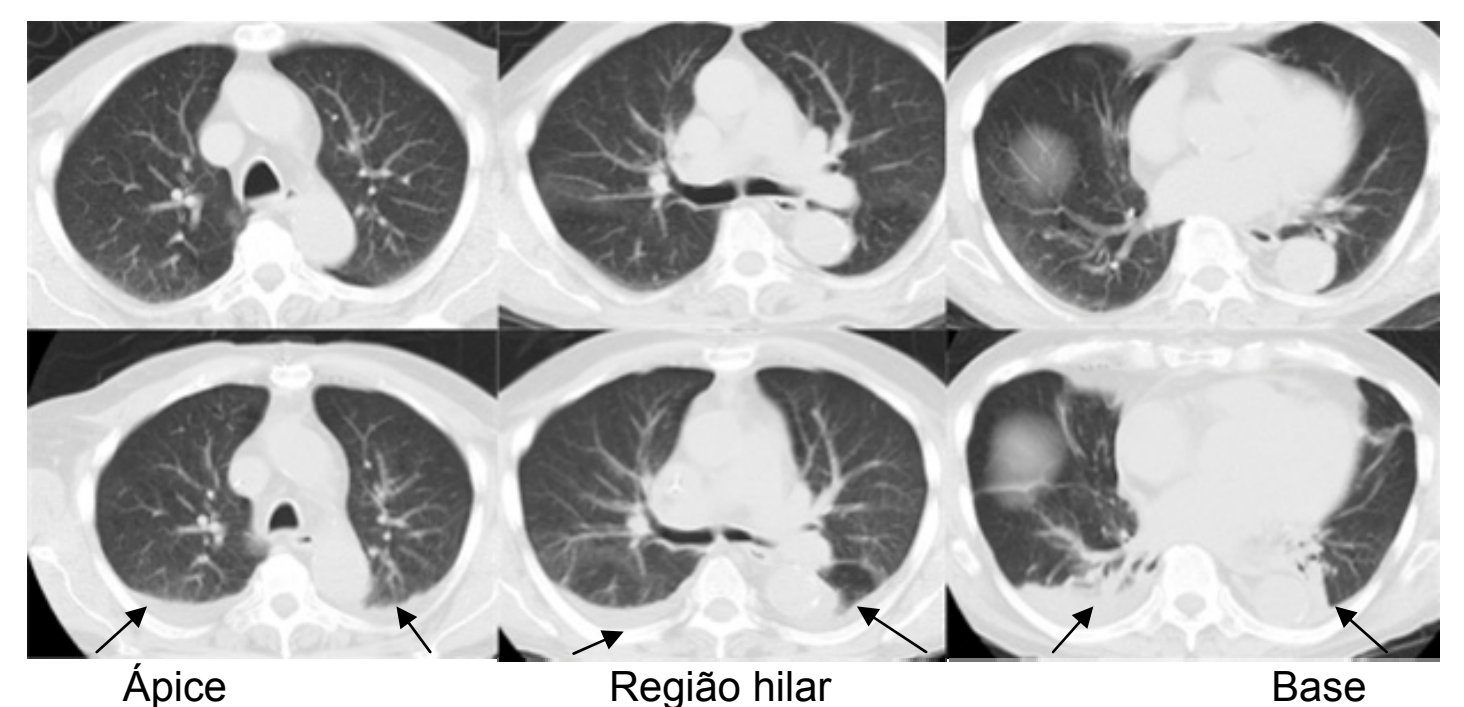

A Figura 4 mostra imagens de TC na altura do ápice, hilo vascular pulmonar e $2 \mathrm{~cm}$ acima do diafragma obtidas no pré-operatório e o primeiro dia pós-operatório. No pré-operatório, foi observado que o volume pulmonar total era $3086 \pm 790 \mathrm{~mL}$, sendo composto por $71 \%$ de gás $(2226 \pm 860 \mathrm{~mL})$, representando a capacidade residual funcional e $29 \%$ de tecidual pulmonar $(862 \pm 180 \mathrm{~mL})$. 
O peso do parênquima pulmonar foi $863 \pm 180$ gramas. Quando o volume do parênquima pulmonar foi avaliado em função da distribuição da aeração, o peso do compartimento de parênquima pulmonar não-aerado foi

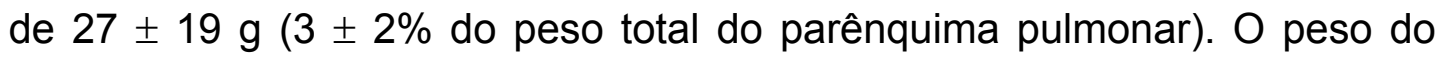
parênquima pulmonar mal-aerado foi de $201 \pm 58 \mathrm{~g}$ (24 $\pm 5 \%$ do peso total do parênquima pulmonar). O peso do parênquima normalmente-aerado foi de $617 \pm 133 \mathrm{~g}$ (72 $\pm 6 \%$ do peso total do parênquima pulmonar).

Como se mostrou na Figura 5, no primeiro dia pós-operatório foi observada uma redução média do volume pulmonar total de $17 \%$, quando comparado com os valores da TC pré-operatórios $(p=0,007)$. Esta diminuição do volume pulmonar total foi secundária a uma redução no volume pulmonar pós-operatório de gás em $31 \%(p<0,001)$, enquanto foi observado um aumento no volume de tecido de 19\% em comparação aos dados pré-operatórios $(\mathrm{p}<0,001)$. 


\section{FIGURA 5}

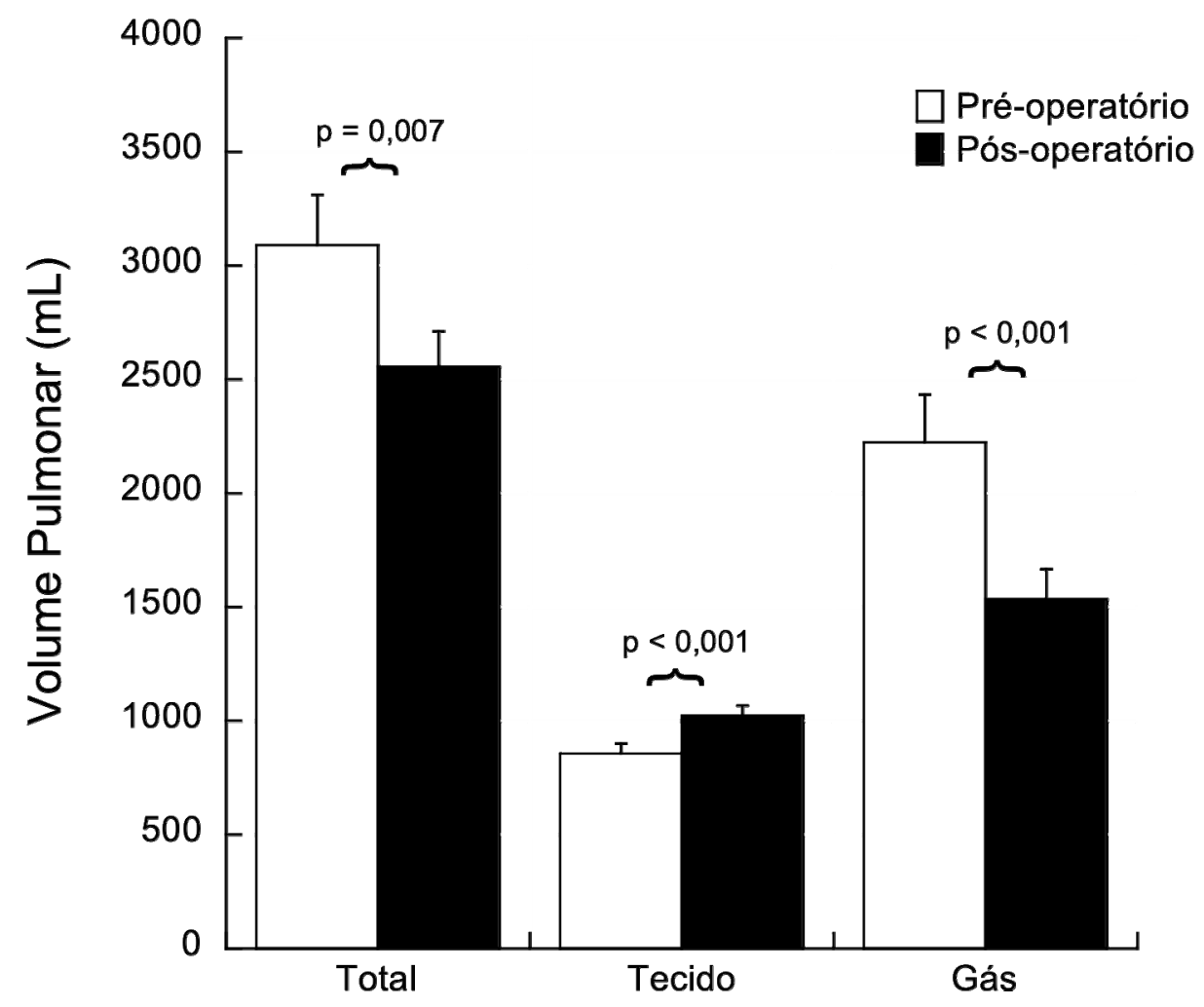

Figura 5: Volume pulmonar total de tecido e gás, computados em reconstruções volumétricas a partir de tomografias computadorizadas de pulmão inteiro no pré-operatório (barras brancas) e no pós-operatório (barras pretas). Dados expressos como média \pm erro padrão. 
Como mostrado na Figura 6, houve um aumento significativo do peso total do parênquima pulmonar no período pós-operatório de 19\% ( $p<$ 0,001). A distribuição relativa do parênquima pulmonar de acordo com a aeração altera-se de maneira significativa no pós-operatório, quando comparada com as tomografias pré-operatórias. Foi observado que o peso global do compartimento de parênquima pulmonar não-aerado aumentou em $253 \pm 97 g(p<0,001)$. Este aumento do compartimento pulmonar colapsado foi observado nos 18 pacientes estudados e compreendia $27,1 \%$ do peso do parênquima pulmonar total. O parênquima pulmonar mal-aerado médio apresentou um pequeno aumento de $72 \pm 68 \mathrm{~g}(p<0,001)$, representando $26,4 \%$ do peso total do parênquima. Por outro lado, o peso do compartimento de parênquima pulmonar normalmente-aerado diminuiu 147 $\pm 119 \mathrm{~g}$ em comparação com os valores pré-operatórios $(p<0,001)$, com este compartimento representando $46,5 \%$ do peso total do parênquima pulmonar. 


\section{FIGURA 6}

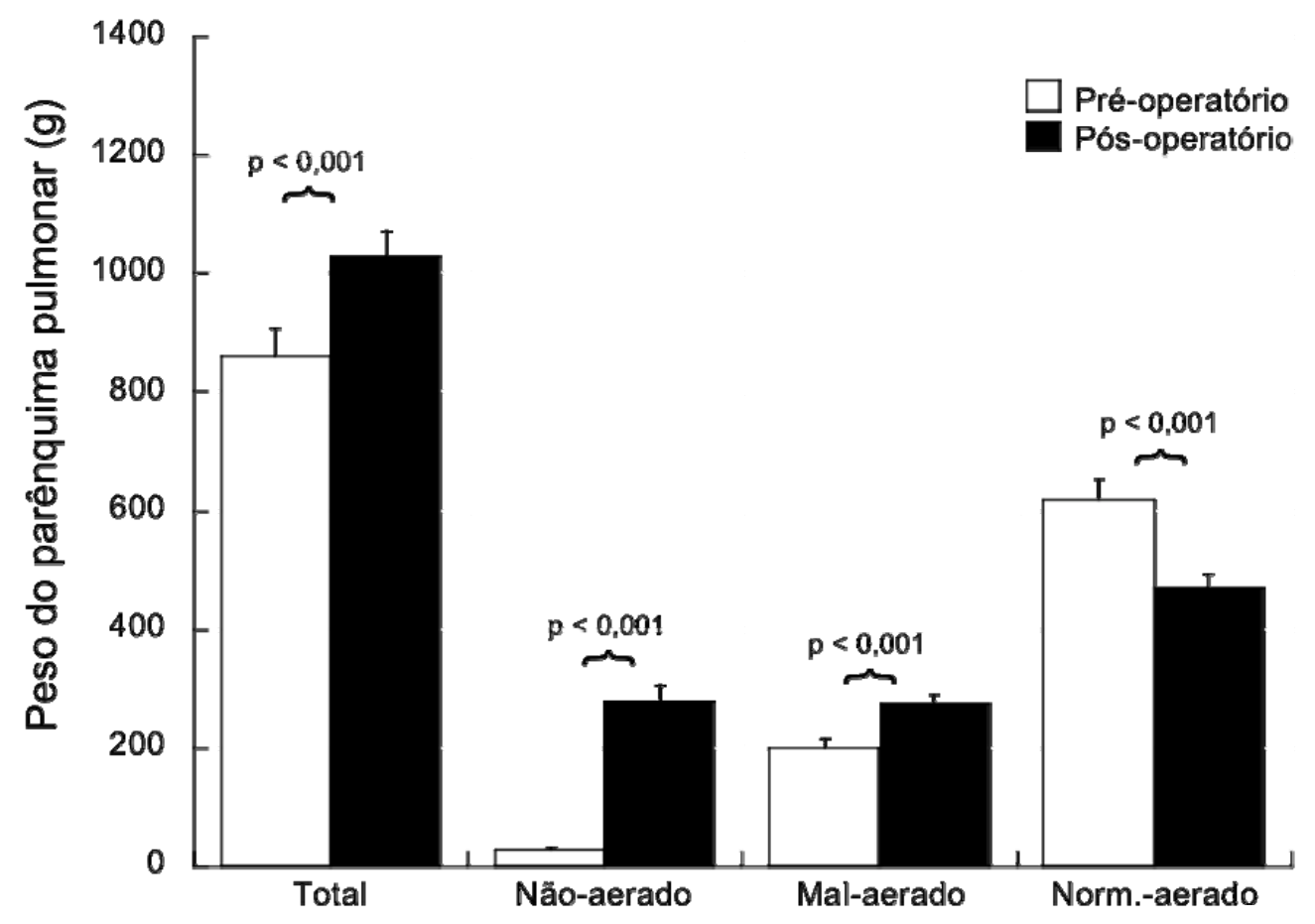

Figura 06: aumento significativo do peso total do parênquima pulmonar no período pós-operatório de $19 \%(p<0,001)$. Quanto à distribuição foi observado que o peso global do compartimento de parênquima pulmonar não-aerado aumentou em $253 \pm 97 \mathrm{~g}(p<0,001)$. O parênquima pulmonar mal-aerado médio apresentou um pequeno aumento de $72 \pm 68 \mathrm{~g}(\mathrm{p}<$ 0,001), representando $26,4 \%$ do peso total do parênquima. $O$ peso do compartimento de parênquima pulmonar normalmente-aerado diminuiu 147 $\pm 119 \mathrm{~g}$ em comparação com os valores pré-operatórios $(p<0,001)$, com este compartimento representando $46,5 \%$ do peso total do parênquima pulmonar. 


\subsection{Correlações entre as alterações observadas na morfologia pulmonar, trocas gasosas e duração da circulação extracorpórea.}

Não foram observadas correlações significativas entre a duração da CEC e a quantidade de parênquima pulmonar não-aerado ou com o aumento do volume de tecido pulmonar. Como foi mostrado na Figura 7, uma correlação significativa negativa foi encontrada entre a redução percentual da fração entre o volume de gás e o volume pulmonar total (redução percentual da $\mathrm{CRF}$ ) e a variação do parênquima pulmonar com aeração reduzida (aumento percentual no peso dos compartimentos nãoaerados e mal-aerado computados conjuntamente). Contudo, nem a variação na fração de parênquima pulmonar não-aerado e nem a variação na fração de parênquima pulmonar mal-aerado foram associadas de maneira independe à redução da CRF.

Nenhuma correlação foi encontrada entre a relação $\mathrm{PaO}_{2} / \mathrm{FiO}_{2}$ calculada no momento da aquisição da TC pós-operatória e o aumento do peso do parênquima pulmonar não-aerado, a diminuição do peso do parênquima pulmonar normalmente-aerado, a variação do parênquima pulmonar com aeração reduzida (- 500 UH a + 200 UH) ou à diminuição percentual da CRF. 


\section{FIGURA 7}

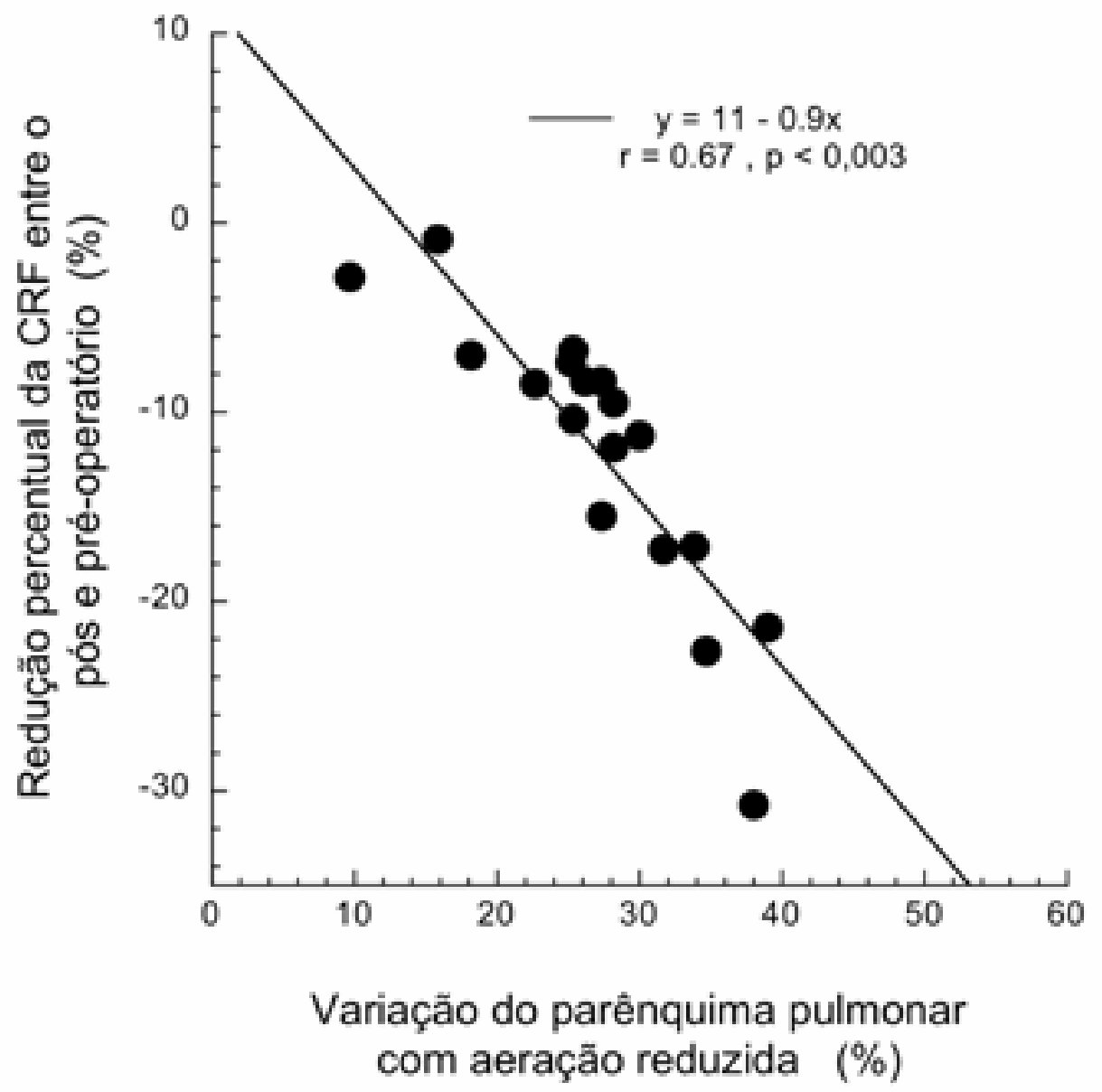

Figura 7: Correlação entre a variação percentual da fração de gás (redução percentual da CRF) e a variação percentual do parênquima pulmonar com aeração reduzida. 
Discussão

(20) 


\section{DiscussÃo}

É de amplo conhecimento que ocorre piora da função pulmonar após cirurgia de revascularização do miocárdio com circulação extracorpórea, caracterizada por desenvolvimento de hipoxemia pósoperatória $^{2}$, mesmo em pacientes sem doença pulmonar pré-operatória ${ }^{4,}$ 14,27. Neste estudo, foi observada uma importante redução na relação $\mathrm{PaO}_{2} / \mathrm{FiO}_{2}$ durante a cirurgia, atingindo seus valores mínimos após a CEC. Apesar do retorno da relação $\mathrm{PaO}_{2} / \mathrm{FiO}_{2}$ para valores próximos aos níveis observados após a indução anestésica no momento 12 horas de pósoperatório, esta permaneceu em torno de $25 \%$ mais baixa que os valores pré-operatórios até a conclusão do estudo, 48 horas após a realização da cirurgia.

O aumento da fração de shunt intrapulmonar secundário às atelectasias tem sido apontado como o principal mecanismo para explicar a piora observada na oxigenação sanguínea durante a cirurgia cardíaca com CEC, tanto no contexto clínico como experimental ${ }^{10,14,19}$. Em diversos estudos, o desenvolvimento de atelectasias nas regiões pulmonares dependentes tem sido consistentemente observado após anestesia geral. Magnusson e colaboradores observaram extensa formação de atelectasias em porcos submetidos à anestesia geral para toracotomia e circulação extracorpórea, representando $35 \%$ da área pulmonar em imagem de tomografia computadorizada justadiafragmática. Contudo, nos animais do 
grupo-controle, submetidos à anestesia geral isolada, sem realização de esternotomia ou circulação extracorpórea, a área de atelectasia representou apenas $1 \%$ da superfície pulmonar. Estes autores relataram uma boa correlação entre a extensão do colapso pulmonar observado na tomografia computadorizada e a fração de shunt intrapulmonar ${ }^{14}$. Hachenberg e colaboradores relataram uma correlação significativa entre a superfície de áreas hiperdensas observadas em tomografias computadorizadas e o shunt intrapulmonar em 11 pacientes evoluindo com disfunção respiratória após cirurgia para revascularização do miocárdio com circulação extracorpórea ${ }^{19}$. Tenling e colaboradores descreveram densidades pulmonares bilaterais em regiões dependentes dos pulmões, que correspondiam a $8 \pm 8 \%$ da área pulmonar total em 3 seções de tomografias computadorizadas torácicas posicionadas $1 \mathrm{~cm}$ acima do diafragma, no hilo pulmonar e no ápice pulmonar em tomografias torácicas obtidas no primeiro dia pós-operatório em 17 de 18 pacientes submetidos à cirurgia para revascularização do miocárdio ou cirurgia para troca de valva mitral. De acordo com estes autores, estas áreas pulmonares hiperdensas correspondiam a $20 \%$ do volume total do parênquima pulmonar. Não encontraram correlação entre a extensão do colapso pulmonar observado em todos os pacientes e a magnitude do shunt intrapulmonar. Contudo, quando foi realizada a análise de subgrupos, uma correlação significativa foi achada entre a extensão das atelectasias e o shunt (rho $=0,95, p<0,01$ ), no subgrupo submetido à cirurgia valvar mitral, mas não no grupo submetido à revascularização miocárdica ${ }^{10}$. 
Neste estudo, foi observado na análise das tomografias computadorizadas torácicas pós-operatórias um aumento em $24 \%$ no peso do parênquima pulmonar colapsado, o que correspondia a $27 \%$ do peso total do parênquima pulmonar. Contudo, não foram observadas correlações entre a extensão das atelectasias quantificadas em todo o parênquima pulmonar e o shunt intrapulmonar ou com a relação $\mathrm{PAO}_{2} / \mathrm{FiO}_{2}$ mensurados 24 horas após a cirurgia, no momento da aquisição da tomografia computadorizada pós-operatória.

Possivelmente, após 24 horas da admissão à unidade de terapia intensiva, outros fatores estão relacionados à piora persistente observada na oxigenação arterial em pacientes submetidos à cirurgia para revascularização do miocárdio em adição à perfusão de regiões pulmonares não aeradas. Usando este método de análise das tomografias computadorizadas torácicas volumétricas, foram observadas alterações na estrutura pulmonar além do aparecimento de extensas atelectasias, e que provavelmente contribuem para a manuntenção desta hipoxemia pósoperatória persistente: 1) importante redução do volume pulmonar de gás em $31 \%$; 2) aumento no peso do parênquima pulmonar, secundário ao aumento de água extravascular pulmonar; 3) redução da massa de parênquima pulmonar normalmente aerado.

O primeiro ponto a ser discutido é que o método utilizado para analisar as estruturas pulmonares nas tomografias computadorizadas torácicas neste estudo é diferente das técnicas utilizadas em estudos prévios avaliando as alterações pulmonares através de tomografia computadorizada 
após cirurgia cardíaca 10,14,19,50,51. Nestes estudos, a quantidade de parênquima pulmonar colapsado foi computada como a área da região pulmonar hiperdensa em um corte tomográfico justadiafragmático ou em três cortes tomográficos no ápice, hilo e bases pulmonares (imagens bidimensionais), que supostamente representam a totalidade do parênquima pulmonar ou pela realização de cálculos baseados no coeficiente CT médio da região de interesse ${ }^{10,14,19,50,51}$. No estudo, foi utilizado o método descrito por Puybasset e colaboradores, que permite a computação do volume pulmonar de gás e de tecido, assim como a distribuição da aeração em cortes tomográficos contíguos e múltiplos obtidos durante curtos períodos de apneia $^{21}$. Utilizando este método, é possível quantificar a massa e volume de parênquima pulmonar colapsado, ao invés de estimá-los a partir de medidas de área de superfície de zonas hiperdensas em uma ou três seções tomográficas. A inferência sobre a quantidade de parênquima pulmonar atelectasiado a partir de superfícies hiperdensas pode ainda hiperestimar a real extensão do colapso pulmonar, por não excluir o volume de gás contido nestas áreas, e que corresponde a $10 \%$ do parênquima pulmonar colapsado. Além do mais, eventos mecânicos, como o deslocamento cefálico do diafragma induzido pela pressão intra-abdominal ${ }^{11,52}$ e a compressão dos lobos inferiores dos pulmões pelo coração ${ }^{53}$, causam elevação regional da pressão pleural nas regiões caudais e dependentes da cavidade torácica, regiões analisadas nas seções tomográficas justadiafragmáticas. Esta elevação regional da pressão pleural promove aumento localizado da extensão do colapso pulmonar, o que poderia limitar a validade da 
quantificação de atelectasias num corte tomográfico basal como representativo de todo o pulmão. Estudando o recrutamento alveolar induzido pela PEEP em pacientes com insuficiência respiratória, Lu e colaboradores observaram que a avaliação do colapso pulmonar em uma seção tomográfica justadiafragmática ou em três seções tomográficas no ápice, hilo e base do pulmão é espuriamente elevada, e não refletia de forma acurada o mesmo evento avaliado em tomografias torácicas volumétricas do pulmão inteiro ${ }^{54}$. Infelizmente, não comparamos a concordância desses métodos intrinsecamente diferentes neste estudo.

Outro mecanismo que poderia explicar a ausência de correlação entre a quantidade de parênquima pulmonar colapsado e a hipoxemia é que a quantificação do colapso pulmonar foi realizada no primeiro dia pósoperatório. Nesse momento, nenhum dos pacientes estava recebendo fármacos anestésicos ou vasoativos capazes de modular o tônus vascular ou impedir o fenômeno de vasoconstrição pulmonar hipóxica (VPH), habitualmente observado em pacientes anestesiados. Seria esperado que a VPF fosse mais efetiva em desviar sangue das regiões atelectasiadas do parênquima pulmonar (hipóxia regional mais intensa) para regiões do parênquima pulmonar caracterizadas por melhor acoplamento da ventilação à perfusão. Esta hipótese é parcialmente suportada pela fraca correlação observada entre o shunt pulmonar e a relação $\mathrm{PaO}_{2} / \mathrm{FiO}_{2}$ computados no momento da aquisição da tomografia pós-operatória. Contudo, não é possível neste estudo prever o comportamento da VPH no momento da 
tomografia computadorizada, sabendo que uma considerável fração de parênquima pulmonar (53,5\%) encontra-se com alteração da ventilação.

Em associação ao aumento do compartimento de parênquima pulmonar não-aerado, foi observado um aumento no compartimento pulmonar pouco-aerado, enquanto a massa de pulmão normalmente-aerada foi reduzida. Apesar de não correlacionado à relação $\mathrm{PaO}_{2} / \mathrm{FiO}_{2}$ observada no momento da tomografia computadorizada pós-operatória, o compartimento de parênquima pulmonar pouco-aerado, onde existe um predomínio de baixa relação ventilação/perfusão ao invés de shunt intrapulmonar verdadeiro, engloba $27 \%$ do parênquima pulmonar. Essa região é perfundida por uma considerável fração do fluxo sanguíneo pulmonar e por isto poderia contribuir para a ineficiência da oxigenação arterial. Experimentalmente, Henzler e colaboradores acharam que a $\mathrm{PAO}_{2}$ era correlacionada ao parênquima pulmonar pouco-aerado, mas não ao compartimento não-aerado em modelo de lesão pulmonar aguda em porcos submetidos à anestesia geral ${ }^{55}$. Em seres humanos, os resultados são conflitantes sobre a influência da extensão do parênquima pulmonar, onde predomina o desacoplamento da relação ventilação/perfusão (mistura venosa) e a oxigenação sanguínea após cirurgia cardíaca. Kjærgaard e colaboradores avaliou o shunt pulmonar e alterações na relação ventilação/perfusão em 14 pacientes após cirurgia cardíaca por meio da construção de curva entre fração inspirada de oxigênio e saturação de oxigênio arterial. Estes autores observaram que os valores de shunt estavam discretamente aumentados e promoviam apenas uma pequena contribuição, 
na maioria dos casos, para a redução da oxigenação sanguínea, enquanto a redução da relação ventilação/perfusão (efeito shunt) tinha um papel preponderante na hipoxemia pós-operatória ${ }^{56}$.

No presente estudo, é possível propor a hipótese de que o aumento na massa de parênquima pouco-aerado contribui para a gênese da hipoxemia pós-operatória pela redução da superfície do parênquima pulmonar normalmente aerado, onde são processadas as trocas gasosas com máxima eficiência. Assim, podemos observar que, neste estudo, houve grande redução na fração do parênquima normalmente-aerado de $72 \%$ para $46 \%$ do parênquima pulmonar total. Contudo, como não foi observada a correlação entre a massa de parênquima pulmonar normalmente-aerado ou a sua variação percentual em relação à TC pré-operatória com a relação $\mathrm{PaO}_{2} / \mathrm{FiO}_{2}$, a redução deste compartimento de parênquima pulmonar não pode ser considerada de maneira isolada como mecanismo crucial, para explicar a piora da oxigenação sanguínea pós-operatória, visto que mecanismos inflamatórios também podem estar envolvidos ${ }^{57}$

Foi observado na tomografia computadorizada pós-operatória um aumento de $2,2 \pm 1,2 \mathrm{~mL} \cdot \mathrm{kg}^{-1}$ no peso total do parênquima pulmonar, secundário a edema pulmonar por permeabilidade vascular aumentada, uma vez que todos os pacientes tinham uma função ventricular esquerda préoperatória normal e as pressões de enchimento foram menores que 18 $\mathrm{mmHg}$ durante todo o estudo. O uso de circulação extracorpórea promove inflamação sistêmica pela exposição dos elementos do sangue à superfície não endotelizada do circuito extracorpóreo e oxigenador de membrana ${ }^{58}$, 
seguido de subsequente aprisionamento de leucócitos na circulação pulmonar ${ }^{59,60}$. O processo inflamatório é agravado pelo fenômeno de isquemia e reperfusão associado à pausa ventilatória e o desvio do fluxo sanguíneo da circulação pulmonar através do circuito extracorpóreo, que resulta em aumento adicional na permeabilidade endotelial pulmonar ${ }^{61}$. Massoudy e colaboradores observaram que 0 aumento da água extravascular pulmonar medido usando o método térmico de 4,8 \pm 0,2 $\mathrm{mL} . \mathrm{kg}^{-1}$ para $6,7 \pm 0,4 \mathrm{~mL} \cdot \mathrm{kg}^{-1}$ em 10 pacientes submetidos à circulação extracorpórea átrio-aórtica, persistiu por pelo menos 4 horas no pósoperatório ${ }^{62}$. Boldt e colaboradores encontraram um aumento significativo, porém transitório, na água extravascular pulmonar computada, medida pela técnica de termodiluição com duplo indicador com verde de indocianina e solução de dextrose gelada em pacientes com função ventricular esquerda normal submetidos à RM com CEC bicaval. De acordo com estes autores, o excesso de água extravascular pulmonar observado na saída de assistência circulatória desapareceu 5 horas após o término da CEC ${ }^{63}$. Diferentemente destes relatos, neste estudo, foi observado que o excesso de água extravascular pulmonar permaneceu no tecido pulmonar pelo menos por 24 horas após a saída de circulação extracorpórea, quando a tomografia computadorizada foi realizada. Estas disparidades entre os diversos estudos são possivelmente relacionadas aos diferentes métodos utilizados para mensurar a água extravascular pulmonar. O método de análise da tomografia computadorizada volumétrica de tórax utilizado neste estudo tem alta acurácia para avaliar água extravascular pulmonar com precisão de 7 
gramas, quando comparado à medida por meio de balança eletrônica ${ }^{64}$. $\mathrm{O}$ edema inflamatório pode contribuir para a gênese da hipoxemia pósoperatória pelo aumento da tendência ao colapso das regiões pulmonares dependentes e pelo peso aumentado do parênquima pulmonar sobrejacente 15,65. Outro mecanismo pelo qual o edema pulmonar poderia contribuir para a piora da oxigenação seria o aumento da distância entre o espaço alveolar e os capilares.

\section{Limitações do método}

Todas as tomografias computadorizadas foram obtidas em pacientes respirando espontaneamente. Apesar do treinamento dos pacientes no sentido de segurarem a respiração após uma expiração normal durante o período de 15 segundos necessários para aquisição da tomografia,não foi possível garantir que todas as imagens tenham sido adquiridas em condições estritas de capacidade residual funcional e, portanto, podem acontecer variações na medida do volume de gás pulmonar, que deveria representar a CRF. Contudo, a maioria dos estudos que avaliou as alterações pulmonares através de tomografia computadorizada de tórax no pós-operatório de cirurgia cardíaca foi realizada em pacientes respirando espontaneamente, além de que a manutenção de sedação nos pacientes para a realização de estudos clínicos seria antiética, e não representaria a realidade. Por tais razões, a obtenção de imagens em pacientes respirando espontaneamente pareceu-nos a técnica mais apropriada. 
Embora o tamanho da população estudada possa permitir a adequada apreciação das alterações na estrutura pulmonar, que ocorrem em pacientes submetidos à cirurgia cardíaca com circulação extracorpórea, o número relativamente pequeno de pacientes está subdimensionado para a detecção de correlações fisiológicas entre as alterações na estrutura pulmonar e a oxigenação sanguínea. 
Conclusões 


\section{CONCLUSÕES}

1) A estrutura pulmonar está profundamente alterada no $1^{\circ}$ dia pós-operatório após revascularização do miocárdio com circulação extracorpórea, caracterizada por aumento significativo das regiões pulmonares não-aeradas e pouco-aeradas, enquanto ocorre a redução do compartimento pulmonar normalmente-aerado. Foi detectada a redução de $31 \%$ no volume de gás pulmonar, enquanto o peso do parênquima pulmonar aumentou em 19\%, secundário a edema pulmonar pós-operatório;

2) Ocorre significativo edema pulmonar inflamatório, que persiste até, pelo menos, o primeiro dia pós-operatório;

3) Não foram observadas correlações entre a extensão das alterações na estrutura pulmonar e as trocas gasosas. 


\section{REFERÊNCIAS}




\section{REFERÊNCIAS}

1. Daganou M, Dimopoulou I, Michalopoulos N, Papadopoulos K, Karakatsani A, Geroulanos S, et al. Respiratory complications after coronary artery bypass surgery with unilateral or bilateral internal mammary artery grafting. Chest. 1998 May;113(5):1285-9.

2. Kolff WJ, Effler DB, Groves LK, Hughes CR, Mc CL. Pulmonary complications of open-heart operations: their pathogenesis and avoidance. Cleve Clin Q. 1958 Apr; 25(2): 65-83.

3. Asimakopoulos G, Smith PL, Ratnatunga CP, Taylor KM. Lung injury and acute respiratory distress syndrome after cardiopulmonary bypass. Ann Thorac Surg. 1999 Sep;68(3):1107-15.

4. Szeles TF, Yoshinaga EM, Alencar W, Brudniewski M, Ferreira FS, Auler Jr JOC, et al. Hypoxemia after myocardial revascularization: Analysis of risk factors. Rev Bras Anestesiol. 2008;58(2):124-36.

5. Vargas FS, Cukier A, Terra-Filho M, Hueb W, Teixeira LR, Light RW. Influence of atelectasis on pulmonary funtion after coronary artery bypass grafting. Chest. 1993 Aug;104(2):434-7. 
6. Vargas FS, Terra-Filho M, Hueb W, Teixeira LR, Cukier A, Light RW, Pulmonary function after coronary artery bypass surgery. Respir Med. 1997 Nov;91(10):629-33.

7. Vargas FS, Uezumi KK, Janete FB, Terra-Filho M, Hued W, Cukier A, et al. Acute pleuropulmonary complications detected by computed tomography following myocardial revascularization. Rev. Hosp Clin Fac Med São Paulo. 2002 Jul-Aug;57(4):135-42.

8. Gale GD, Teasdale SJ, Sanders DE, Bradwell PJ, Russell A, Solaric B. Pulmonary atelectasis and other respiratory complications after cardiopulmonary bypass and investigation of aetiological factors. Can Anaesth Soc J. 1979 Jan;26(1):15-21.

9. Lindberg P, Gunnarsson L, Tokics L, Secher E, Lundquist H, Brismar B, et al. Atelectasis and lung function in the postoperative period. Acta Anaesthesiol Scand. 1992 Aug;36(6):546-53.

10. Tenling A, Hachenberg T, Tyden H, Wegenius G, Hedenstierna G. Atelectasis and gas exchange after cardiac surgery. Anesthesiology. 1998 Aug;89(2):371-8.

11. Froese $A B$, Bryan AC. Effects of anesthesia and paralysis on diaphragmatic mechanics in man. Anesthesiology. 1974;41:242-54. 
12. Pelosi P, D'andrea L, Vitale G, Pesenti A, Gattinoni L. Vertical gradient of regional lung inflation in adult respiratory distress syndrome. Am J Respir Crit Care Med. 1994;149(1):8-13.

13. Rothen HU, Sporre B, Engberg G, Wegenius G, Hogman M, Hedenstierna G. Influence of gas composition on recurrence of atelectasis after a reexpansion maneuver during general anesthesia. Anesthesiology. 1995 Apr;82(4):832-42.

14. Magnusson L, Zemgulis V, Wicky S, Tyden H, Thelin S, Hedenstierna G. Atelectasis is a major cause of hypoxemia and shunt after cardiopulmonary bypass: an experimental study. Anesthesiology. 1997 Nov;87(5):1153-63.

15. Singh NP, Vargas FS, Cukier A, Terra-Filho M, Teixeira LR, Light RW. Arterial blood gases after coronary artery bypass surgery. Chest. 1992 Nov;102(5):1337-41.

16. Nozawa E, Kobayashi E, Matsumoto ME, Feltrim MI, Carmona MJ, Auler JJ. Assessment of factors that influence weaning from long-term mechanical ventilation after cardiac surgery. Arq Bras Cardiol. 2003 Mar;80(3):301-10.

17. Marshall BE. Importance of hypoxic pulmonary vasoconstriction with atelectasis. Adv Shock Res. 1982;8:1-12. 
18. Villar J, Blazquez MA, Lubillo S, Quintana J, Manzano JL. Pulmonary hypertension in acute respiratory failure. Crit Care Med. 1989 Jun;17(6):523-6.

19. Hachenberg T, Brussel T, Roos N, Lenzen H, Mollhoff T, Gockel B, et al. Gas exchange impairment and pulmonary densities after cardiac surgery. Acta Anaesthesiol Scand. 1992 Nov;36(8):800-5.

20. Hedenstierna G. Atelectasis formation and gas exchange impairment during anaesthesia. Monaldi Arch Chest Dis. 1994 Sep;49(4):315-22.

21. Puybasset L, Cluzel P, Chao N, Slutsky AS, Coriat P, Rouby JJ, et al. A computed tomography scan assessment of regional lung volume in acute lung injury. Am J Respir Crit Care Med. 1998;158(5 Pt 1):164455.

22. Yeden S,Wunderik R: Causes of prolonged mechanical ventilation after coronary artery bypass surgery. Chest. 2002; 122: 245-252.

23. Weissmann C: Pulmonary complications after cardiac surgery. Sem cardiothor Vasc Anesth. 2004; 8:185-213.

24. Møller JT, Wittrup M, Johansen SH. Hypoxemia in the postanesthesia care unit: an observer study. Anesthesiology 1990; 73: 890-895. 
25. Reeder MK, Goldman MD, Loh L et al. Postoperative hypoxaemia after major abdominal vascular surgery. Br J Anaesth 1992; 68: 23-26.

26. Breiman RS, Beck JW, Korobkin M, Glenny R, Akwari OE, Heaston DK, Moore AV, Ram PC. Volume determinations using computed tomography. Am J Roentgenol. 1982; 138: 329-33.

27. Tagliabue M, Casella TC, Zincone GE, Fumagalli R, Salvini E. CT and chest radiography in the evaluation of adult respiratory distress syndrome. Acta Radiol. 1994; 35:230-4.

28. Hedenstierna G. Gas exchange during anaesthesia. $\mathrm{Br} J$ Anaesth. 1990; 64: 507-514.

29. Gattinoni I, Pesenti A, Bombino M, Baglioni S, Rivolta M, Rossi F, Rossi G, Fumagalli R, Marcolin R, Mascheroni D et al. Relationships between lung computed tomographic density, gas exchange, and PEEP in acute respiratory failure. Anesthesiology. 1988; 69:824-32.

30. Gattinoni L, Pelosi P, Vitale G, Pesenti AL, DA, Mascheroni D. Body position changes redistribute lung computed-tomographic density in patients with acute respiratory failure. Anesthesiology. 1991; 74:15-23. 
31. Gattinoni L, Pesenti A. The concept of baby lung. Intensive. Care Medicine. 2005; 31(6): 776-784.

32. Amato MBP, Barbas CSV, Medeiros DM, Magaldi RB, Schettino GPP, Lorenzi-Filho G, Kairalla RA, Deheinzelin D, Munoz C, Oliveira R, Takagaki TY, Carvalho CRR. Effect of a protective-ventilation strategy on mortality in the acute respiratory distress syndrome. The New England Journal of Medicine. 1998; 338(6): 347-54.

33. Ventilation with lower tidal volumes as compared withtraditional tidal volumes for acute lung injury and the acute respiratory distress syndrome. The acute respiratory distress syndrome networkthe. New England Journal of Medicine. 342 (18): 1301-08

34. Terragni PP, Rosboch G, Tealdi A, Corno E, Menaldo E, Davini O, Gandini G, Herrmann P, Mascia L, Quintel M, Slutsky AS, Gattinoni L, and Ranieri MV. Tidal Hyperinflation during Low Tidal Volume Ventilation in Acute Respiratory Distress Syndrome. American Journal of Respiratory and Critical Care Medicine. 2007; 175:160-6.

35. Lachmann B. Open up the lung and keep the lung open. Intensive Care Med 1992;18:319 -21 
36. Hachenberg T, Tenling A, Nystrom SO, et al. Ventilation perfusion equality in patients undergoing cardiac surgery. Anesthesiology. 1994; $80: 509-19$

37. Odenstedt H, Aneman A, Karason S, et al. Acute hemodynamic change during lung recruitment in lavage and endotoxin induced ALI. Intensive Care Med. 2005; 31:112-20.

38. Hedenstierna G, Strandberg A, Brismar B et al. - Functional residual capacity, thoracoabdominal dimensions, and central blood volume during general anesthesia with muscle paralysis and mechanical ventilation. Anesthesiology. $1985 ; 62: 247-54$

39. Hedenstierna G. Brismar B, Strandberg A et al. - New aspects on atelectasis during anaesthesia. Clin Physiol. 1985;5 Suppl 3:127-31.

40. Cabrera MR, Nakamura GE, Montague DA, Cole RP. Effect of airway pressure on pericardial pressure. Am Rev Respir Dis. 1989; 140:65967.

41. Lundh R, Hedenstierna G. Ventilation-perfusion relationships during anaesthesia and abdominal surgery. Acta Anaesthesiol Scand 1983; 27: 167-73. 
42. O'Donohue W.J, Baker JP. Controlled Low-Flow Oxygen in the Management of Acute Respiratory Failure; Chest. 1973; 63:818-20

43. Robinson JD, Lupkiewicz SM, Palenik L, Lopez LM, Ariet M. Determination of ideal body weight for drug dosage calculations. Am J Hosp 1983;40:1016-9.

44. Goepfert MSG, Reuter DA. Akyol D, Lamm P, Kilger E; Goetz EA. Goaldirected fluid management reduces vasopressor and catecholamine use in cardiac surgery patients. Intensive Care Medicine. 2007; 33(1).

45. Davies AR, Bellomo R, Raman JS, Gutteridge GA, Buxton BF. High lactate predicts the failure of intraaortic balloon pumping after cardiac surgery. Ann Thorac Surg. 2001; 71:1415-20.

46. Puybasset L, Gusman P, Muller JC, Cluzel P, Coriat P, Rouby JJ. Regional distribution of gas and tissue in acute respiratory distress syndrome. III. Consequences for the effects of positive end-expiratory pressure. CT Scan ARDS Study Group. Adult Respiratory Distress Syndrome. Intensive Care Med. 2000; 26: 1215-27.

47. Vieira SR, Puybasset L, Richecoeur J, Lu Q, Cluzel P, Gusman PB, et al. A lung computed tomographic assessment of positive end-expiratory pressure-induced lung overdistension. Am J Respir Crit Care Med. 1998; 158: 1644-1655 
48. Vieira SR, Puybasset L, Lu Q, Richecoeur J, Cluzel P, Coriat P, et al. A scanographic assessment of pulmonary morphology in acute lung injury. Significance of the lower inflection point detected on the lung pressure-volume curve. Am J Respir Crit Care Med. 1999 May;159 (5 Pt 1):1612-23.

49. Mull RT. Mass estimates by computed tomography: physical density from CT numbers. Am J Roentgenol. 1984 Nov;143(5):1101-4.

50. Hachenberg T, Lundquist H, Tokics L, Brismar B, Hedenstierna G. Analysis of lung density by computed tomography before and during general anaesthesia. Acta Anaesthesiol Scand. 1993 Aug;37(6):549-55.

51. Magnusson L, Zemgulis V, Tenling A, Wernlund J, Tyden H, Thelin S, et al. Use of a vital capacity maneuver to prevent atelectasis after cardiopulmonary bypass: an experimental study. Anesthesiology. 1998; 88(1):134-42.

52. Malbrain ML, Chiumello D, Pelosi P, Bihari D, Innes R, Ranieri VM, et al. Incidence and prognosis of intraabdominal hypertension in a mixed population of critically ill patients: a multiple-center epidemiological study. Crit Care Med. 2005 Feb; 33(2):315-22. 
53. Malbouisson LM, Busch CJ, Puybasset L, Lu Q, Cluzel P, Rouby JJ. Role of the heart in the loss of aeration characterizing lower lobes in acute respiratory distress syndrome. CT Scan ARDS Study Group. Am J Respir Crit Care Med. 2000 Jun;161(6):2005-12.

54. Lu Q, Malbouisson LM, Mourgeon E, Goldstein I, Coriat P, Rouby JJ. Assessment of PEEP-induced reopening of collapsed lung regions in acute lung injury: are one or three CT sections representative of the entire lung? Intensive Care Med. 2001 Sep;27(9):1504-10.

55. Henzler D, Pelosi P, Dembinski R, Ullmann A, Mahnken A, Rossaint R, et al. Respiratory compliance but not gas exchange correlates with changes in lung aeration after a recruitment maneuver: an experimental study in pigs with saline lavage lung injury. Critical Care. 2005; 9(5):R471 - R82.

56. Kjaergaard S, Rees SE, Gronlund J, Nielsen EM, Lambert P, Thorgaard P, et al. Hypoxaemia after cardiac surgery: clinical application of a model of pulmonary gas exchange. Eur J Anaesthesiol. 2004 Apr; 21(4):296-301

57. Guillermo L, Roberto C, Jorge U. Renal preservation in cardiac surgeryCurrent Opinion in Anaesthesiology: February 1998 - Volume 11 - Issue 1 - pp 9-13 
58. de Vroege R, Rutten PM, Kalkman C, Out TA, Jansen PG, Eijsman L, et al. Biocompatibility of three different membrane oxygenators: effects on complement, neutrophil and monocyte activation. Perfusion. 1997 Nov;12(6):369-75.

59. Massoudy P, Zahler S, Becker BF, Braun SL, Barankay A, Richter JA, et al. Significant leukocyte and platelet retention during pulmonary passage after declamping of the aorta in CABG patients. Eur $J$ Med Res. 1999 May 26; 4(5):178-82.

60. Massoudy P, Zahler S, Becker BF, Braun SL, Barankay A, Meisner H. Evidence for inflammatory responses of the lungs during coronary artery bypass grafting with cardiopulmonary bypass. Chest. 2001 Jan; 119(1):31-6.

61. Schlensak C, Doenst T, Beyersdorf F. Lung ischemia during cardiopulmonary bypass. Ann Thorac Surg. 2000 Jul; 70(1): 337-8.

62. Massoudy P, Piotrowski JA, van de Wal HC, Giebler R, Marggraf G, Peters J, et al. Perfusing and ventilating the patient's lungs during bypass ameliorates the increase in extravascular thermal volume after coronary bypass grafting. Ann Thorac Surg. 2003 Aug; 76(2): 516-21; discussion 21-2. 
63. Boldt J, Zickmann B, Dapper F, Hempelmann G. Does the technique of cardiopulmonary bypass affect lung water content? Eur $J$ Cardiothorac Surg. 1991;5(1):22-6.

64. Malbouisson LM, Preteux F, Puybasset L, Grenier P, Coriat P, Rouby JJ. Validation of a software designed for computed tomographic (CT) measurement of lung water. Intensive Care Med. 2001 Mar; 27(3): 6028.

65. Auler-Junior JO, Saldiva PH. Pulmonary structure and extravascular lung water after cardiopulmonary bypass. Braz J Med Biol Res. $1986 ; 19(6): 707-14$ 